\title{
Further ALMA observations and detailed modeling of the Red Rectangle
}

\author{
V. Bujarrabal ${ }^{1}$, A. Castro-Carrizo ${ }^{2}$, J. Alcolea ${ }^{3}$, M. Santander-García ${ }^{3,4}$, H. van Winckel $^{5}$, and C. Sánchez Contreras ${ }^{6}$ \\ 1 Observatorio Astronómico Nacional (OAN-IGN), Apartado 112, 28803 Alcalá de Henares, Spain \\ e-mail: v.bujarrabal@oan.es \\ 2 Institut de Radioastronomie Millimétrique, 300 rue de la piscine, 38406 Saint-Martin-d'Hères, France \\ 3 Observatorio Astronómico Nacional (OAN-IGN), C/ Alfonso XII, 3, 28014 Madrid, Spain \\ 4 Instituto de Ciencia de Materiales de Madrid (CSIC), Calle Sor Juana Inés de la Cruz 3, 28049 Cantoblanco, Madrid, Spain \\ 5 Instituut voor Sterrenkunde, K.U. Leuven, Celestijnenlaan 200B, 3001 Leuven, Belgium \\ 6 Centro de Astrobiología (CSIC-INTA), ESAC Campus, 28691 Villanueva de la Cañada, Madrid, Spain
}

Received 18 March 2016 / Accepted 7 June 2016

\begin{abstract}
Aims. We aim to study the rotating and expanding gas in the Red Rectangle, which is a well known bipolar nebula surrounding a double stellar system whose primary is a post-asymptotic giant branch (post-AGB) star. We analyze the properties of both components and the relation between them. Rotating disks have been very elusive in post-AGB nebulae, in which gas is almost always found to be in expansion.

Methods. We present new high-quality ALMA observations of this source in $\mathrm{C}^{17} \mathrm{O} J=6-5$ and $\mathrm{H}^{13} \mathrm{CN} J=4-3$ line emission and results from a new reduction of already published ${ }^{13} \mathrm{CO} J=3-2$ data. A detailed model fitting of all the molecular line data, also discussing previous maps and single-dish observations of lines of CO, CII, and CI, was performed using a sophisticated code that includes an accurate nonlocal treatment of radiative transfer in 2D (assuming axial symmetry). These observations (of low- and highopacity lines requiring various degrees of excitation) and the corresponding modeling allowed us to deepen the analysis of the nebular properties. We also stress the uncertainties, particularly in the determination of the boundaries of the CO-rich gas and some properties of the outflow.

Results. We confirm the presence of a rotating equatorial disk and an outflow, which is mainly formed of gas leaving the disk. The mass of the disk is $\sim 0.01 M_{\odot}$, and that of the CO-rich outflow is around ten times smaller. High temperatures of $\gtrsim 100 \mathrm{~K}$ are derived for most components. From comparison of the mass values, we roughly estimate the lifetime of the rotating disk, which is found to be of about $10000 \mathrm{yr}$. Taking data of a few other post-AGB composite nebulae into account, we find that the lifetimes of disks around post-AGB stars typically range between about 5000 and more than $20000 \mathrm{yr}$. The angular momentum of the disk is found to be high, $\sim 9 M_{\odot} \mathrm{AU} \mathrm{km} \mathrm{s}{ }^{-1}$, which is comparable to that of the stellar system at present. Our observations of $\mathrm{H}^{13} \mathrm{CN}$ show a particularly wide velocity dispersion and indicate that this molecule is only abundant in the inner Keplerian disk, at $\lesssim 60$ AU from the stellar system. We suggest that HCN is formed in a dense photodissociation region (PDR) due to the UV excess known to be produced by the stellar system, following chemical mechanisms that are well established for interstellar medium PDRs and disks orbiting young stars. We further suggest that this UV excess could lead to an efficient formation and excitation of PAHs and other C-bearing macromolecules, whose emission is very intense in the optical counterpart.
\end{abstract}

Key words. stars: AGB and post-AGB - circumstellar matter - radio lines: stars - planetary nebulae: individual: Red Rectangle

\section{Introduction}

Most planetary and preplanetary nebulae (PNe, PPNe) show conspicuous departures from spherical symmetry, often with a clear axis of symmetry. In addition, very massive $\left(\sim 0.1 M_{\odot}\right)$ and fast (20-200 $\left.\mathrm{km} \mathrm{s}^{-1}\right)$ bipolar outflows are characteristic of PPNe and thought to be crucial to understand the formation and shaping of PNe; see, e.g., Bujarrabal et al. (2001), Balick \& Frank (2002), and Sánchez Contreras \& Sahai (2012). On the other hand, circumstellar envelopes around asymptotic giant branch (AGB) stars, from which PNe have evolved, are in general spherical and expand isotropically at moderate velocities $\sim 10-15 \mathrm{~km} \mathrm{~s}^{-1}$; it is thought that their ejection is powered by radiation pressure. The linear momentum carried by the postAGB outflows is too high to be powered by momentum transfer from stellar photons, which strengthens the need of some other mechanism to explain the dynamics in this phase. It is often assumed that this spectacular evolution is driven by magnetocentrifugal launching, as probably occurs in forming stars, which implies that rotating disks must be systematically formed from previously ejected material (e.g., Soker 2001; Frank \& Blackman 2004). The presence of a stellar or substellar companion is necessary in nebulae around evolved stars, since otherwise the ejected shells do not have enough angular momentum to form such disks.

However, in most planetary and preplanetary nebulae only gas in expansion is detected (Bujarrabal et al. 2001; Sánchez Contreras \& Sahai 2012, etc.) and the disks postulated to orbit post-AGB stars have been very elusive. To date, the Keplerian velocity field has been unambiguously identified by means of interferometric mm-wave maps of CO line emission in only two of these putative disks: in the Red Rectangle (Bujarrabal et al. 2005, 2013b) and AC Her (Bujarrabal et al. 2015). 
The Red Rectangle and AC Her belong to a class of binary post-AGB stars with low-mass nebulae and indications of compact disks (e.g., van Winckel 2003); about 100 post-AGB objects in our Galaxy are classified into this wide class of objects. These objects are characterized by their spectral energy distributions (SEDs), which particularly shows a near-infrared (NIR) excess indicating hot dust close to the stellar system. This indeed suggests a stable structure, as the stars are no longer in a state of copious mass loss (De Ruyter et al. 2006; Gezer et al. 2015). The inner dust around a few of these post-AGB stars has been resolved with optical interferometry confirming the very compact nature of the emitting region (Deroo et al. 2006; Hillen et al. 2015, 2016). Additional indications of the longevity of these disks come from studies of the high degree of dust processing (e.g., Gielen et al. 2011). Single-dish observations of ${ }^{12} \mathrm{CO}$ and ${ }^{13} \mathrm{CO} \mathrm{mm}$-wave emission in a sample of these post-AGB stars systematically yielded characteristic line profiles, with a prominent single or double peak and moderate-velocity wings, which are strikingly similar to those of the Red Rectangle and AC Her (Bujarrabal et al. 2013a). As discussed in that work, profiles of this kind are also found in disks around young stars (particularly in T Tauri variables), and have been proven, both from theoretical and observational grounds, to be very reliable indicators of rotating disks. It was proposed that Keplerian disks are relatively widespread in post-AGB nebulae, at least in those surrounding this class of binary stars.

The Red Rectangle is the prototype and best-studied example of this wide class of post-AGB nebulae. It consists of an equatorial disk orbiting a double stellar system, with a period of about 320 days, plus a beautiful X-shaped axisymmetric nebula in expansion that is seen in visible wavelengths (e.g., Men'shchikov et al. 2002; Cohen et al. 2004). The nebula equator and axis of symmetry are easily identifiable in the optical images; the axis is slightly out of the plane of the sky (by about $5^{\circ}$ ) and its projection in the plane of the sky shows a PA $\sim 10-15 \mathrm{deg}$. Men'shchikov et al. deduced a distance of $710 \mathrm{pc}$ for this source, which is compatible with our previous analysis of CO data.

The first maps of the $\mathrm{CO}$ mm-wave emission from the rotating disk in the Red Rectangle were obtained with the Plateau de Bure Interferometer (PdBI; Bujarrabal et al. 2005). The Red Rectangle was also observed in sub-mm line and continuum emissions using ALMA. Maps of ${ }^{12} \mathrm{CO} J=3-2$ and $J=6-5$ and ${ }^{13} \mathrm{CO} J=3-2$ were presented by Bujarrabal et al. (2013b), together with a simplified modeling of the data. The rotation of the equatorial disk was conspicuous in those maps. The positionvelocity diagrams along the direction of the equator, $\mathrm{PA} \sim 102.5$, clearly show the signature of Keplerian rotation. In the ALMA maps by Bujarrabal et al. (2013b), the disk appears as an equatorial feature at low LSR velocities within $\pm 2.5 \mathrm{~km} \mathrm{~s}^{-1}$. Molecular gas in slow expansion was tentatively detected in the PdBI and single-dish data and spectacularly confirmed by the ALMA observations, corresponding to the high-latitude $\mathrm{CO}$ emission in the maps at moderate velocities, which is more or less coincident with the optical image. This composite structure was confirmed by comparison with nebula models containing both gas in rotation and expansion (Bujarrabal et al. 2013b). The diameter of the detected disk is $\sim 5 \times 10^{16} \mathrm{~cm}\left(\sim 5^{\prime \prime}\right)$ and the extent of the CO-rich outflow is slightly larger. In view of the structure and velocity of the expanding component, which seems in some way an extension of the rotating disk and roughly occupies the region between the disk and the X-shaped optical image, the outflowing gas seems to be extracted from the disk. Such an ejection could be due to interaction with the axial jet that is active in this object (Witt et al. 2009; Thomas et al. 2013) or to low-velocity magnetocentrifugal launching from the extended disk, a process that takes place in certain disks around young stars (Ferreira et al. 2006; Panoglou et al. 2012, etc.). In addition, single-dish data of the Red Rectangle in various transitions of ${ }^{12} \mathrm{CO}$ and ${ }^{13} \mathrm{CO}$ (up to $J=16-15$ ) also exist and have been analyzed using sophisticated nonlocal treatments of radiative transfer and line excitation (Bujarrabal \& Alcolea 2013).

As mentioned, another similar source, AC Her, was later confirmed to show a Keplerian disk; remarkably, no sign of expansion was found in this source. Molecular gas in expansion has been found from direct mapping of $\mathrm{CO}$ emission in two of these objects: 89 Her (Bujarrabal et al. 2007) and IRAS 19125+0343 (unpublished PdBI observations). Rotation was probably present in the detected central condensation, but the velocity field was not angularly resolved and those disks must be significantly smaller than for the Red Rectangle.

The CO emission maps show that the optical X-shaped structure in the Red Rectangle very probably represents the inner surface of the biconical (outflowing) component that emits in $\mathrm{CO}$ lines, although the optical image is significantly more extended than the detected CO-rich gas. This optical X-like feature is composed of emission of PAHs (polycyclic aromatic hydrocarbons) and ERE features (extended red emission; also probably due to carbonaceous macromolecules), while scattered light or atomic emission contribute negligibly to this feature; see Cohen et al. (2004). C-bearing macromolecules must be particularly abundant in this conical structure, although the disk dust is silicaterich. This property was invoked to conclude that the central star recently changed from $\mathrm{O}$ - to $\mathrm{C}$-rich owing to nuclear processing in the stellar interior (Waters et al. 1998) or to changes in abundances due to reaccretion of circumstellar material after dust formation (van Winckel 2014). As we show in what follows, the different composition of both components could also be due to the development of a PDR in the inner disk.

In this paper, we present unpublished ALMA data of $\mathrm{C}^{17} \mathrm{O} J=6-5$ and $\mathrm{H}^{13} \mathrm{CN} J=4-3$ emission, together with a new analysis of some of the previously published ALMA maps and a detailed modeling of the whole set of observations of (light) molecules using the sophisticated code presented in a previous paper (Bujarrabal \& Alcolea 2013). Some Herschel observations of CII and CI far-infrared (FIR) lines are also discussed in comparison with the emission of $\mathrm{C}^{17} \mathrm{O}$ and $\mathrm{H}^{13} \mathrm{CN}$.

\section{Observations}

\subsection{New ALMA observations and new reduction of the ${ }^{13} \mathrm{CO} \mathrm{J}=3-2$ maps}

We observed the Red Rectangle with ALMA $^{1}$ using receiver bands 7 and 9 in October-November 2012. Details on the observations and data reduction can be found in Bujarrabal et al. (2013b). Maps of ${ }^{12} \mathrm{CO}$ and ${ }^{13} \mathrm{CO} J=3-2$ emission (at 0.8 mm, ALMA band 7) and of ${ }^{12} \mathrm{CO} J=6-5$ (at $0.4 \mathrm{~mm}$, ALMA band 9) were already published (Bujarrabal et al. 2013b). Here we present observations of the $\mathrm{H}^{13} \mathrm{CN} J=4-3$ line (band 7) and the $\mathrm{C}^{17} \mathrm{O} J=6-5$ line (band 9), obtained during the same observational runs, as well as a new image synthesis for the ${ }^{13} \mathrm{CO} J=3-2$ emission with higher signal-to-noise $(\mathrm{S} / \mathrm{N})$ ratio, which allows a deeper analysis of the outflowing gas.

\footnotetext{
1 ALMA is a partnership of ESO (representing its member states), NSF (USA) and NINS (Japan), together with NRC (Canada) and NSC and ASIAA (Taiwan), in cooperation with the Republic of Chile. The Joint ALMA Observatory is operated by ESO, AUI/NRAO and NAOJ. We made use of the ALMA dataset ADS/JAO.ALMA\#2011.0.00223.S.
} 
In band 9 data and the maps of the weak $\mathrm{H}^{13} \mathrm{CN}$ line, we used natural weighting to improve the mapping quality. In our new reduction of the ${ }^{13} \mathrm{CO} J=3-2$ emission, we also used natural weighting. The half-power beamwidth (HPBW) in band 7 data is of about 0 ". $59 \times 0$ '. 56 for natural weighting. A HPBW resolution of $\sim 0$.' $31 \times 0$.' 25 was obtained in band 9 . Our new results are shown in Figs. 1, 3, and 5 (Figs. 2, 4, and 6 show, respectively, our best model fits of those maps; see Sect. 5). In these figures we also show a Hubble Space Telescope (HST) optical image (see Bujarrabal et al. 2013b, for details on the comparison of both images). In all cases the (logarithmic) contour spacing is given both in units of Jy/beam and $\mathrm{K}$ (Rayleigh-Jeans equivalent brightness temperature); we corrected a minor error in the conversion between $\mathrm{K}$ and $\mathrm{Jy} /$ beam given in our previous work for the ${ }^{13} \mathrm{CO} J=3-2$ maps due to confusion between natural and robust beam widths. As shown in Bujarrabal et al. (2013b), continuum emission was detected and resolved, with a total flux of $\sim 0.6 \mathrm{Jy}$ in band 7 and of $\sim 3.5 \mathrm{Jy}$ in band 9 . To best analyze the weak line emission, the continuum emission was subtracted from all the channel maps here presented.

\subsection{Herschel/HIFI observations of atomic lines in the Red Rectangle}

The HIFI heterodyne spectrometer on board the Herschel Space Telescope (Pilbratt et al. 2010; de Graauw et al. 2010) was used to observe three carbon transitions. The data were taken in double side-band (DSB) fast-chop mode, and using the $\mathrm{H}$ and $\mathrm{V}$ receivers, which were averaged once ruling out significant differences in the observed lines with both receivers.

The CI $J=1-0$ line $(492.161 \mathrm{GHz})$ was observed on September 07, 2012, in the 1a band, with a spectral resolution of $0.61 \mathrm{~km} \mathrm{~s}^{-1}$. The CI $J=2-1$ line $(809.344 \mathrm{GHz})$ was observed on March 23, 2013, in the 3a band and with a spectral resolution of $0.37 \mathrm{~km} \mathrm{~s}^{-1}$. Finally, CII $J=2-1(1901.280 \mathrm{GHz})$ was secured on March 20, 2013, in the $7 \mathrm{~b}$ band, with a spectral resolution of $0.32 \mathrm{~km} \mathrm{~s}^{-1}$. The beam sizes were $43.1,26.2$, and 11.2 arcsec, respectively.

We reduced the data with the standard HIFI pipeline in the HIPE software with a modified version of the level 2 algorithm that yields unaveraged spectra with all spectrometer sub-bands stitched together. The spectra were then exported to CLASS using the hiClass tool within HIPE, for further inspection. Data suffering from significant ripple residuals were filtered and discarded and, in each case, the remaining spectra were averaged. Baseline removal of degree 2 was applied to the final spectra. The data were originally calibrated in antenna temperature units and later converted into main-beam temperatures $\left(T_{\mathrm{mb}}\right)$ and flux units; see more details in, for example, Alcolea et al. (2013).

\subsection{Flux lost by interferometric over-resolution}

Given the very high resolution of our ALMA data and that some of the components we detect, notably the outflow, are relatively extended, we can expect that a fraction of the flux can be lost in the interferometric process (particularly in these Cycle-0 observations). It is sometimes difficult to estimate the amount of lost flux, which depends on the coverage of the interferometric visibilities in the $u v$ plane, on the calibration accuracy, and on the intrinsic extent and intensity of the brightness distribution. However, the amount of lost flux is very relevant in this paper because model predictions are compared with maps and singledish observations of various lines. Single-dish data are available for the ${ }^{12} \mathrm{CO} J=6-5$ line, observed with Herschel/HIFI; see Bujarrabal \& Alcolea (2013). The angle-integrated flux profile obtained from our ${ }^{12} \mathrm{CO} J=6-5$ map reaches a peak of $70 \mathrm{Jy}$, therefore the flux recovered by the ALMA maps is typically about $60-70 \%$ of the total flux (see Fig. A.1). This factor is reasonable, mainly for $\mathrm{mm}$ or sub-mm interferometric observations, and strongly suggests that the representation of the nebula shape by means of our ALMA maps is reliable. The percentage of lost flux is higher in the line wings, for LSR velocities $\sim \pm 5-8 \mathrm{~km} \mathrm{~s}^{-1}$, in which as much as $\sim 60 \%$ of the total emission can be lost. This is likely resulting from the relatively wide and weak emission from the outflow, and we expect it to be noticeable even at lower frequencies. The lost flux is similar in the very center of the line, $V_{\mathrm{lsr}} \sim 0 \mathrm{~km} \mathrm{~s}^{-1}$, probably because of the extended emission coming from the outflowing gas placed in the plane of the sky and from the outermost disk regions.

Since the distributions of the $\mathrm{C}^{17} \mathrm{O}$ and $\mathrm{H}^{13} \mathrm{CN}$ emissions are significantly more compact than that of ${ }^{12} \mathrm{CO} J=6-5$, a very moderate amount of flux is expected to be filtered out in these cases. ${ }^{13} \mathrm{CO} J=3-2$ emission is also relatively compact and should show low over-resolution effects, except, perhaps, for the weak outflow emission at intermediate velocities. We also expect a moderate flux loss for the intense ${ }^{12} \mathrm{CO} J=3-2$ line (maps presented in Bujarrabal et al. 2013b) because its extent/resolution ratio is smaller than for ${ }^{12} \mathrm{CO} J=6-5$ and the calibration conditions are better for its lower frequency. The comparison with the single-dish spectrum published by De Beck et al. (2010) is not straightforward because of the uncertain conversion to flux in Jy, but our conclusions seem confirmed with a low flux loss in the central part of the profile and a moderate loss of about $25-30 \%$ in the line wings.

In our data fitting, we try to take into account that the fraction of lost flux is not always negligible. As seen below, we attempt to reach a compromise between the fittings of the maps and the total intensity obtained from single-dish data.

\section{Line emission model}

We modeled our ALMA maps using a sophisticated and accurate code, which simultaneously calculates the molecular level populations and solves the radiative transfer equations for the geometry and kinematics of the Red Rectangle. The code was first presented in Bujarrabal \& Alcolea (2013), which includes the details of the numerical treatment and exhaustive tests of the calculation accuracy (in particular for the case of $\mathrm{CO}$ ). Our model assumes a 2D gas cloud showing axial symmetry. Radiative transfer is solved with a fully nonlocal treatment and the molecular level populations are calculated in a high number of cells. The level populations in each point depend on the intensity of radiation arriving at the frequencies of the various transitions and, at the same time, the radiative transfer at these frequencies depends on the populations of the levels joined by the transitions. Therefore, both the calculations of the level populations and radiation intensity are performed simultaneous and coherently, in an iterative process that involves radiative coupling between the different parts of the cloud. Collisional transitions are also taken into account in each cell, and collisional rates were taken from the LAMBDA database ${ }^{2}$.

Finally, once convergence has been attained, the radiative transfer equations are solved in the direction of the observer. In Bujarrabal \& Alcolea (2013), the resulting brightness distribution in the plane of the sky is convolved with single-dish, wide

\footnotetext{
2 http://WWW.strw.leidenuniv.nl/ moldata
} 


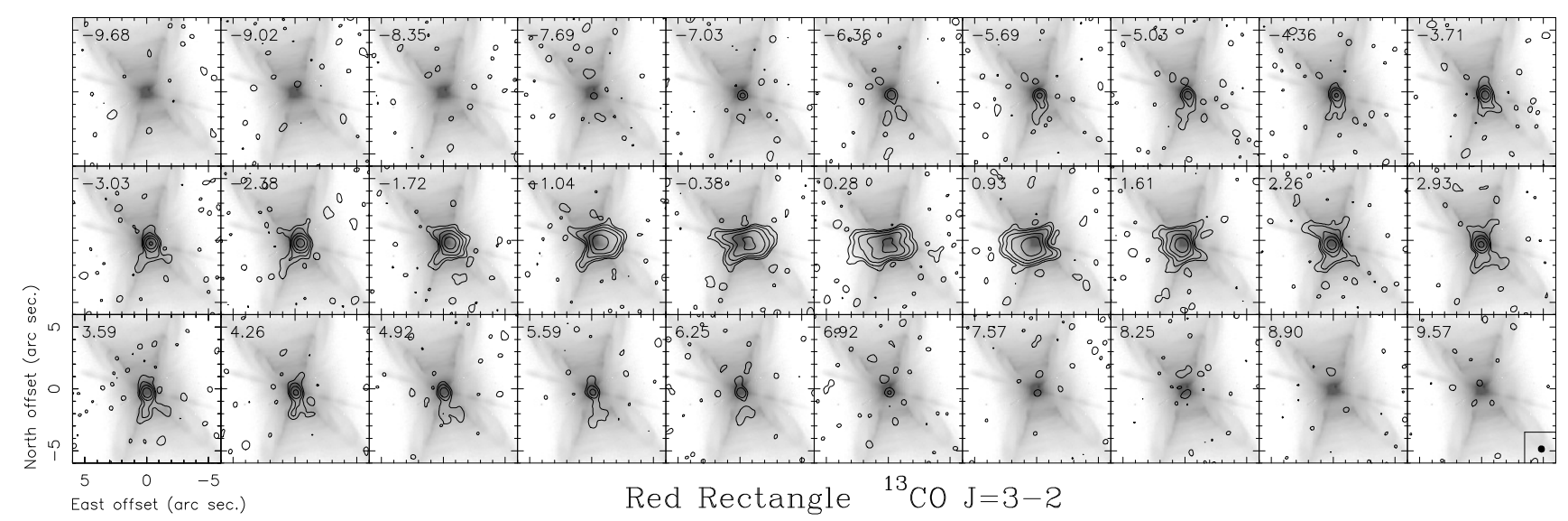

Fig. 1. ALMA ${ }^{13} \mathrm{CO} J=3-2$ observations of the Red Rectangle. To better show the contribution of the various components, these maps were obtained using natural weighting in the image deconvolution and a velocity resolution of $0.66 \mathrm{~km} \mathrm{~s}^{-1}$. The resulting beam size (HPBW) is 0 "' $59 \times 0 .{ }^{\prime \prime} 56$ (shown in the insert). The contours are $-0.01,0.01,0.03,0.09,0.27$, and $0.81 \mathrm{Jy} /$ beam (logarithmic spacing by a factor 3 , equivalent to $0.34,1.02$, $3.06,9.18$, and $27.5 \mathrm{~K}$ ). We note the large contrast between expanding gas emission and the peak of the equatorial disk brightness, almost by a factor 100. The HST optical image is also shown. As in most maps shown in this paper, we subtracted the continuum.

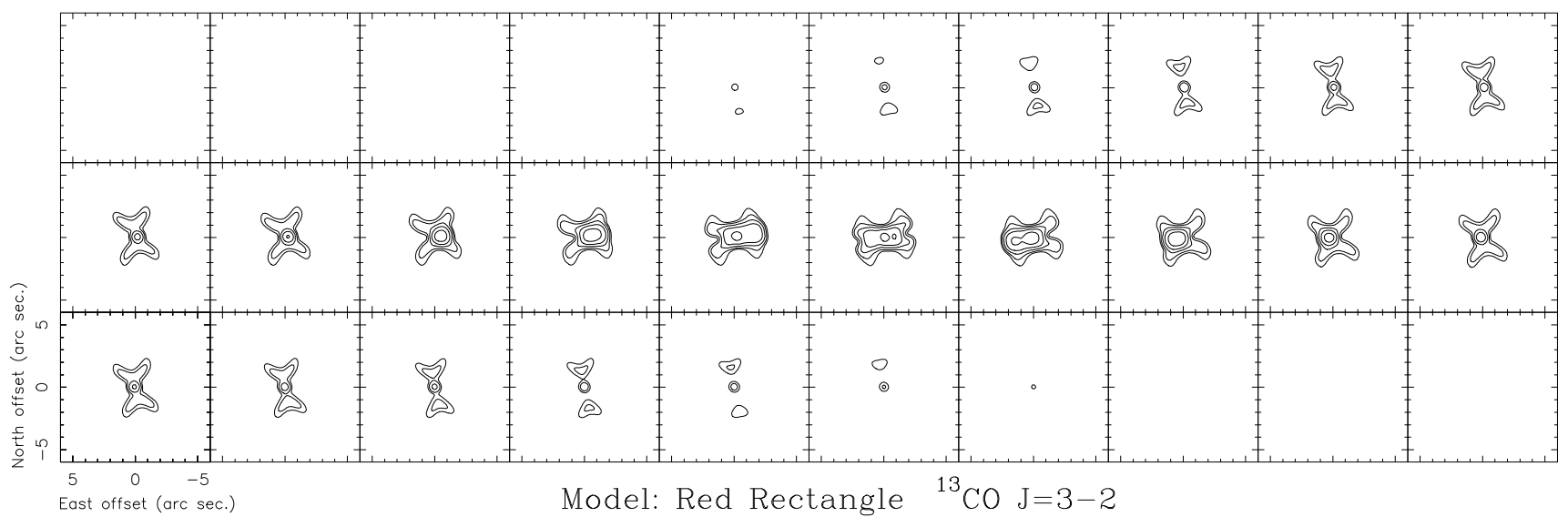

Fig. 2. Theoretical maps of ${ }^{13} \mathrm{CO} J=3-2$ obtained for our best-fit model, to be compared with ALMA maps, Fig. 1. The contours and angular and velocity units are the same as for the observational data.

beams, yielding spectral profiles of the whole emission of the source. Here, the brightness distribution is convolved with the interferometric beam to obtain synthetic maps directly comparable to the observed maps. The beam convolution and calculation of synthetic maps are similar to those shown in Bujarrabal et al. (2013b).

In the preliminary analysis performed by Bujarrabal et al. (2013b), the molecular excitation treatment was much simpler, just assuming LTE excitation. That approach may be valid for low- $J$ transitions of ${ }^{12} \mathrm{CO}$, for which the low Einstein coefficients and high optical depth guarantee thermalization under general conditions. In the calculations performed in this paper, including $C O$ high- $J$ levels, optically thin lines, and $\mathrm{H}^{13} \mathrm{CN}$ transitions, we cannot assume LTE. In addition, a local treatment of excitation, such as in large velocity gradient (LVG) or LVG-like approximations, is very useful when one deals with expanding gas, but cannot be applied to rotating disks, in which regions placed at large distances (comparable in fact to the disk size) and with very different physical conditions radiatively interact. A complex, nonlocal treatment of radiative transfer is therefore necessary in our case.

In the treatment of the $\mathrm{H}^{13} \mathrm{CN}$ line excitation, we do not include the hyperfine structure of the rotational levels. No effects of the partial overlap between components on radiative transfer and level population is expected in the very optically thin rotational lines. In the observed $J=4-3$ line, the main components are separated by $\sim 0.1 \mathrm{~km} \mathrm{~s}^{-1}$, and the satellite components are at least ten times weaker. The effects of the $J=4-3$ splitting in the line profile and the calculation of the synthetic maps are, in comparison with the moderate $\mathrm{S} / \mathrm{N}$ observations, negligible.

\section{Observational results from our ALMA maps}

Our ALMA maps of ${ }^{12} \mathrm{CO} J=3-2$ and $J=6-5$ emission from the Red Rectangle were presented in Bujarrabal et al. (2013b). In that work, we also show maps of ${ }^{13} \mathrm{CO} J=3-2$; to better show the weak emission of this line from the expanding gas far from the equator, we performed a new image synthesis of those data, assuming natural weighting of the visibilities (which yields a wider beam and a better sensitivity) and degrading the spectral resolution to $0.66 \mathrm{~km} \mathrm{~s}^{-1}$. As in the rest of the images shown here, we subtracted the continuum because of the weak line brightness (see ALMA data of the dust continuum emission in Bujarrabal et al. 2013b). The resulting ${ }^{13} \mathrm{CO} J=3-2$ maps are shown in Fig. 1, in which we can see the weak emission from the outflow (the slight extension to high latitudes farther than $1^{\prime \prime}$ and following the X-shaped optical image, mostly noticeable at 


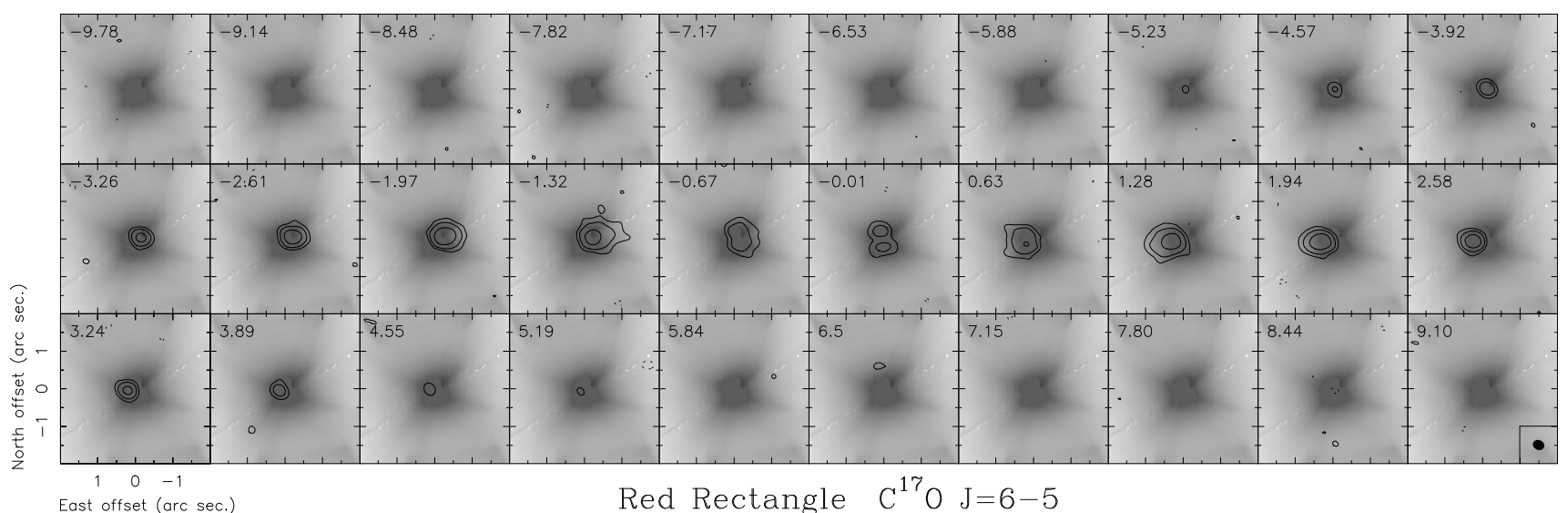

Fig. 3. ALMA $\mathrm{C}^{17} \mathrm{O} J=6-5$ observations of the Red Rectangle. The resulting beam size (HPBW) is 0 .' $^{\prime} 31 \times 0$ "' 26 (shown in the insert) and the chosen velocity resolution is $0.45 \mathrm{~km} \mathrm{~s}^{-1}$. The contours are $-0.075,0.075,0.225$, and $0.675 \mathrm{Jy} / \mathrm{beam}$ (logarithmic spacing by a factor 3 , equivalent to $2.54,7.62$, and $22.9 \mathrm{~K}$ ). The emission is weak compared with the ${ }^{12} \mathrm{CO} J=6-5$ emission shown in Bujarrabal et al. (2013b) and Fig. B.1, which reaches a peak of $\sim 200 \mathrm{~K}$. The continuum has been subtracted.

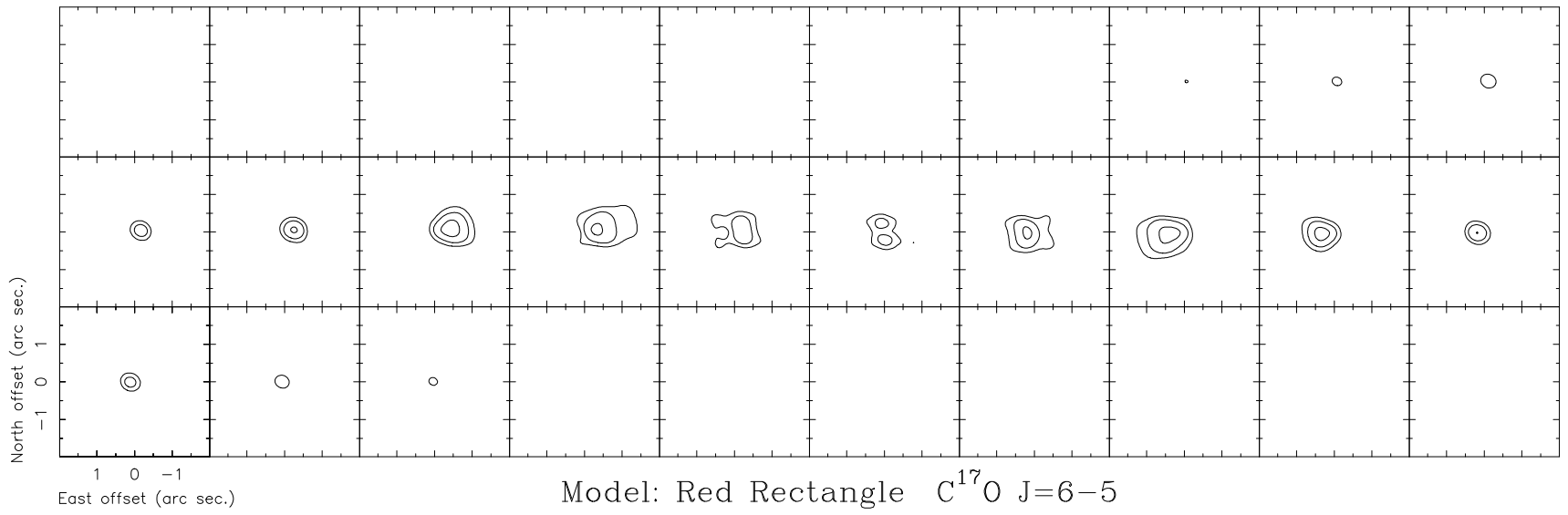

Fig. 4. Theoretical maps of $\mathrm{C}^{17} \mathrm{O} J=6-5$ obtained for our best-fit model for comparison with the ALMA maps in Fig. 3. The contours and angular and velocity units are the same as for the observational data.

intermediate velocities, $\pm 2-6 \mathrm{~km} \mathrm{~s}^{-1}$ ), almost 100 times weaker than the brightness peak.

In Fig. 3, we show our maps of $\mathrm{C}^{17} \mathrm{O} J=6-5$. As expected, the emission is relatively weak compared with the ${ }^{12} \mathrm{CO} J=6-5$ maps shown in Bujarrabal et al. (2013b) and Fig. B.1, which reach a peak of $\sim 200 \mathrm{~K}$, pointing out that $\mathrm{C}^{17} \mathrm{O} J=6-5$ is very probably optically thin. The outflow emission, in particular, is found to be very faint in $\mathrm{C}^{17} \mathrm{O} J=6-5$, at least 60 times weaker than the peak. Meanwhile, the measured contrast in ${ }^{12} \mathrm{CO} J=6-5$ is just a factor $\sim 10$.

The $\mathrm{H}^{13} \mathrm{CN} J=4-3$ maps are shown in Fig. 5. This is by far the weakest molecular line detected in the Red Rectangle, this is the only map in our observations with just a moderate $\mathrm{S} / \mathrm{N}$, of about 20 . The brightness levels, $\lesssim 1 \mathrm{~K}$, are very low for a source that reaches a brightness of $\sim 200 \mathrm{~K}$ in ${ }^{12} \mathrm{CO} J=6-5$ and kinetic temperatures of several hundred Kelvin in inner regions (see Sect. 5). The very broad line with maxima at $V_{\mathrm{lsr}} \sim \pm 4-5 \mathrm{~km} \mathrm{~s}^{-1}$ is to be compared with the emission of the other optically thin line, $\mathrm{C}^{17} \mathrm{O} J=6-5$, with maxima at about $\pm 2 \mathrm{~km} \mathrm{~s}^{-1}$. No trace of outflow emission is found in our $\mathrm{H}^{13} \mathrm{CN} J=4-3$ maps with a brightness $\lesssim 7 \mathrm{mJy} /$ beam, which is more than $\sim 10$ times weaker than the peak.

Further representations of our observations are shown in Figs. 10, A.2, and A.3, which show in particular positionvelocity $(\mathrm{P}-\mathrm{V})$ diagrams along different cuts. We note the conspicuous Keplerian patterns in cuts along the equator (Fig. A.2 and cut H0O in Figs. 10 and A.3) and the tentative detection of slow rotation in gas outside the disk $(\mathrm{H} 1 \mathrm{~N}, \mathrm{H} 1 \mathrm{~S}$, H2N, H2S in Figs. 10 and A.3; see Sect. 6). We also note the disk velocity pattern in cuts perpendicular to the equator (inner $1^{\prime \prime}$ in cuts V1 and V2, Figs. 10 and A.3), which suggests that the rotation velocity mostly depends on the distance to the center, not on the distance to the axis.

\section{Model fitting}

We used our numerical code (Sect. 3) to perform model fitting of all maps obtained with ALMA in the Red Rectangle, namely of the lines ${ }^{12} \mathrm{CO} J=3-2$ and $J=6-5$ (Figs. 1 and 3 of Bujarrabal et al. 2013b and Fig. B.1), ${ }^{13} \mathrm{CO} J=3-2$ (Fig. 1), $\mathrm{C}^{17} \mathrm{O} J=6-5$ (Fig. 3), and $\mathrm{H}^{13} \mathrm{CN} J=4-3$ (Fig. 5). We also reproduced the ${ }^{12} \mathrm{CO}$ and ${ }^{13} \mathrm{CO}$ single-dish profiles presented in Bujarrabal \& Alcolea (2013). As we discussed in Sect. 2.3, the fraction of lost flux by over-resolution in the interferometric observations is small, but not always negligible, particularly in the line wings coming from the outflow. In our fitting process, we try to fit both the total intensity obtained from single-dish data and the maps, keeping in mind that the brightness distributions of the mapped lines can be somewhat overestimated by our model calculations 


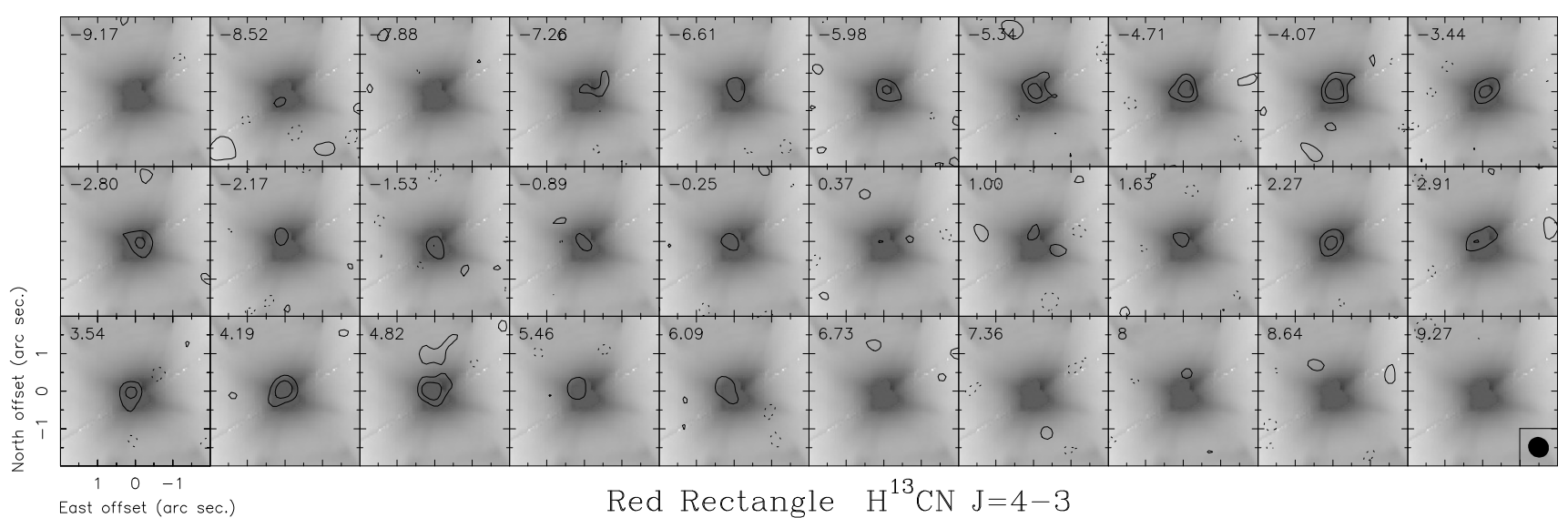

Fig. 5. ALMA $\mathrm{H}^{13} \mathrm{CN} J=4-3$ observations of the Red Rectangle. The resulting beam size (HPBW) is $0 .{ }^{\prime \prime} 57 \times 00^{\prime \prime} 53$ (shown in the insert). The contours are $-0.01,0.01,0.02$, and $0.04 \mathrm{Jy} /$ beam (logarithmic spacing by a factor 2 , equivalent to $0.34,0.68,1.36 \mathrm{~K}$ ). In this figure, the contours are separated by a factor 2 . This is, by far, the weakest molecular line detected in the Red Rectangle. The continuum has been subtracted.

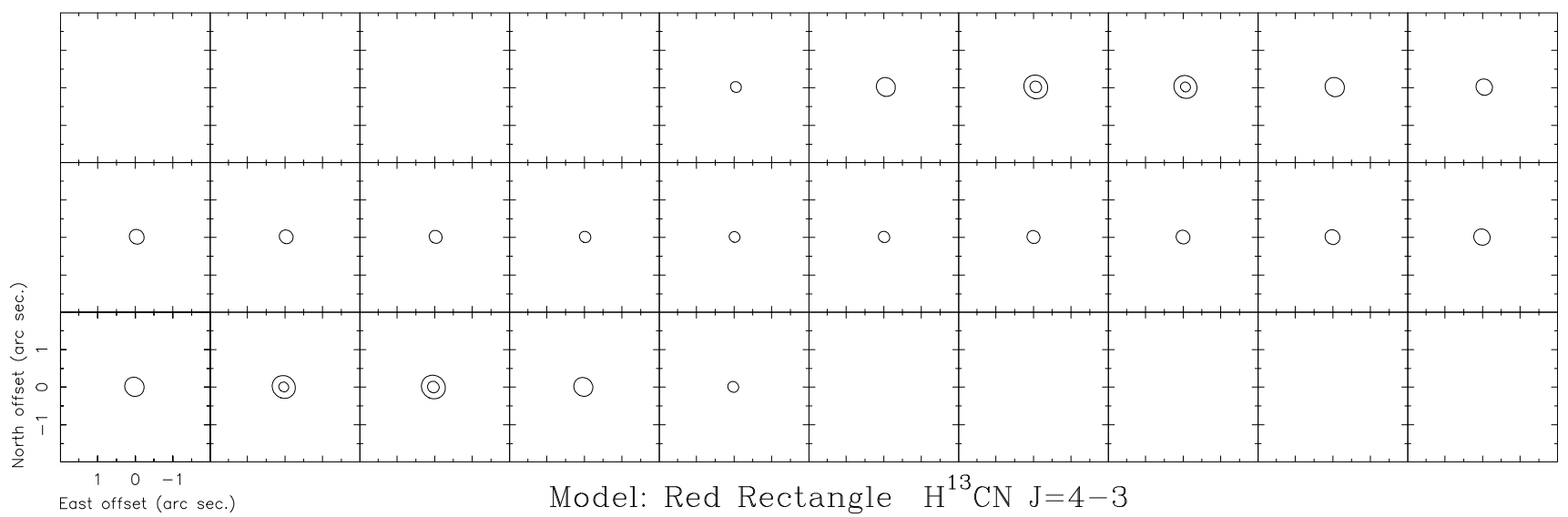

Fig. 6. Theoretical maps of $\mathrm{H}^{13} \mathrm{CN} J=4-3$ obtained for our best-fit model for comparison with ALMA maps in Fig. 5. The contours and angular and velocity units are the same as for the observational data.

with respect to the observations, especially for the velocities at which the lost flux is larger.

The synthetic maps from our best-fit model are shown in Fig. B. 2 for the ${ }^{12} \mathrm{CO} J=6-5$ (that was not fitted in our previous paper, see observational data reproduced in Fig. B.1), Fig. 2 for the new reduction of ${ }^{13} \mathrm{CO} J=3-2$ data, Fig. 4 for $\mathrm{C}^{17} \mathrm{O} J=6-5$, and Fig. 6 for $\mathrm{H}^{13} \mathrm{CN} J=4-3$. In Fig. 7 we present the new fitting of the single-dish observations. Other synthetic maps, to help our discussion in the following sections on the nebula properties, are shown in Appendix B.

\subsection{Fitting of $\mathrm{CO}$ line maps}

A representation of the structure, velocity field and density distribution in our model nebula is shown in Fig. 8. In general, the deduced nebular properties are very similar to those presented in our previous works. The emitting regions are slightly smaller to account for the smaller extent found in observations with higher resolution and in optically-thin lines. In principle, the new data are the best representation of the actual shape and the new model should be more accurate, but we must keep in mind the flux lost in those lines, which may lead to some underestimates of the size in the present work. The shape of the expanding component is also somewhat different, yielding a slightly better fitting and intuitively accounting for the idea that the $\mathrm{CO}$ extent in this diffuse component must be limited by photodissociation. The total outflow extent is roughly compatible with photodissociation calculations by the interstellar UV field (Mamon et al. 1988), which indicates that CO-rich diffuse-enough gas expanding at about $10 \mathrm{~km} \mathrm{~s}^{-1}$ extends just a few $10^{16} \mathrm{~cm}$. In any case, the dissociation process in our complex source is more difficult to describe than for the calculations performed by Mamon et al. for a spherically symmetric AGB shell expanding at constant radial velocity. It is in particular difficult to be sure about the starting point and past kinetics of the outflowing gas and the dust density in our source. Although, as mentioned, the new convex shape tends to reproduce the observations at long distances from the center slightly better, the exact shape of the cloud boundary is uncertain, since it has just a moderate effect on the model predictions. Therefore, we do not attempt here to derive, from model fitting or other considerations, an exact shape of the outer boundary of the CO-rich outflow, and we just adopt that extent as indicative of the possible real extent as it is possibly a lower limit.

The laws and parameters describing the physical conditions in the different components of our best-fit CO-emission model are given in Table 1 (and complemented with the graphical description in Fig. 8). As we can see in our synthetic maps (Figs. 2, 4,6 ) and single-dish data fitting (Fig. 7), the data are correctly reproduced. From the density and size we derive, we find a total disk mass of $\sim 1.3 \times 10^{-2} M_{\odot}$. The mass of the outflow is found to be $\sim 1.2 \times 10^{-3} M_{\odot}$. These values are about twice those found from the general analysis by Bujarrabal et al. (2013a), 


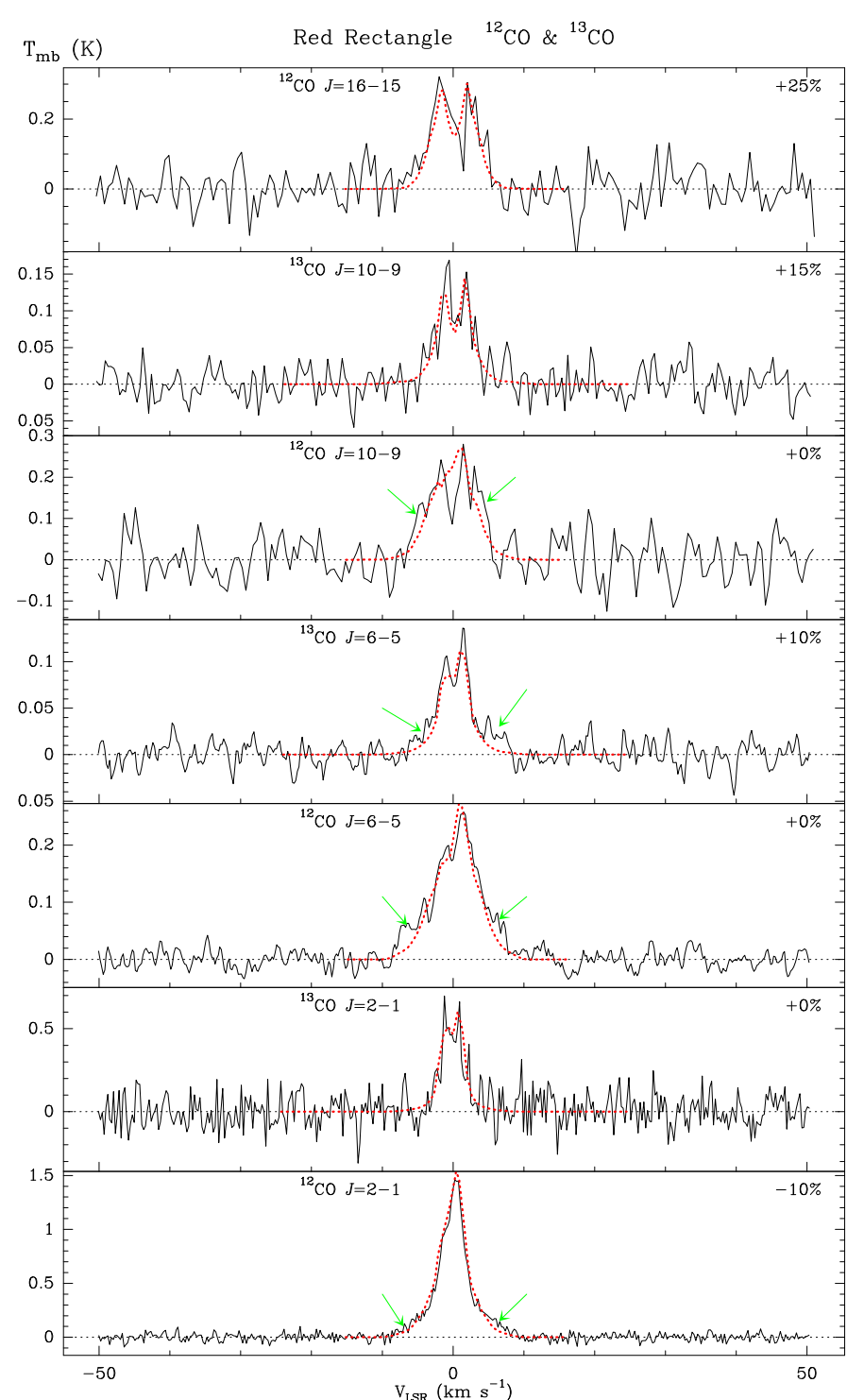

Fig. 7. Observed CO line profiles of the mm and FIR transitions (black solid line) and the predictions of our code (red dotted line); see Sect. 5.2. The line-wing emission of some lines is somewhat underestimated (green arrows). The values of the free scale parameter applied to the model predictions that account for uncertainties in the calibration are indicated in the upper right corners.

but well within the usual uncertainty in the determination of the parameter. The discrepancy mainly comes from the lower $\mathrm{CO}$ abundances deduced here, which are $X\left({ }^{12} \mathrm{CO}\right) \sim 1.2 \times 10^{-4}$ and $X\left({ }^{13} \mathrm{CO}\right) \sim 1.2 \times 10^{-5}$. This value of the $\mathrm{CO}$ abundance is low, but it is still in the range of what is found in post-AGB nebulae with $X\left({ }^{13} \mathrm{CO}\right)$ typically ranging between 1 and $2 \times 10^{-5}$ (Bujarrabal et al. 2001, 2015; Santander-García et al. 2012; etc). The angular momentum of our best-fit model disk is of about $2.7 \times 10^{52} \mathrm{~g} \mathrm{~cm}^{2} \mathrm{~s}^{-1}$, i.e. $\sim 9.1 M_{\odot} \mathrm{AU} \mathrm{km} \mathrm{s}{ }^{-1}$. We recall that the analysis of lines requiring different degrees of excitation and showing different opacities allows us to distinguish the effects on the line intensity of the different parameters, in particular, of the density and relative abundance; see some discussion on the uncertainties of the derived values in Sect. 5.4.

In the comparison between the observed and predicted maps, we sometimes find a certain overestimation of the outflow emission, particularly in the predicted emission of the outflow in

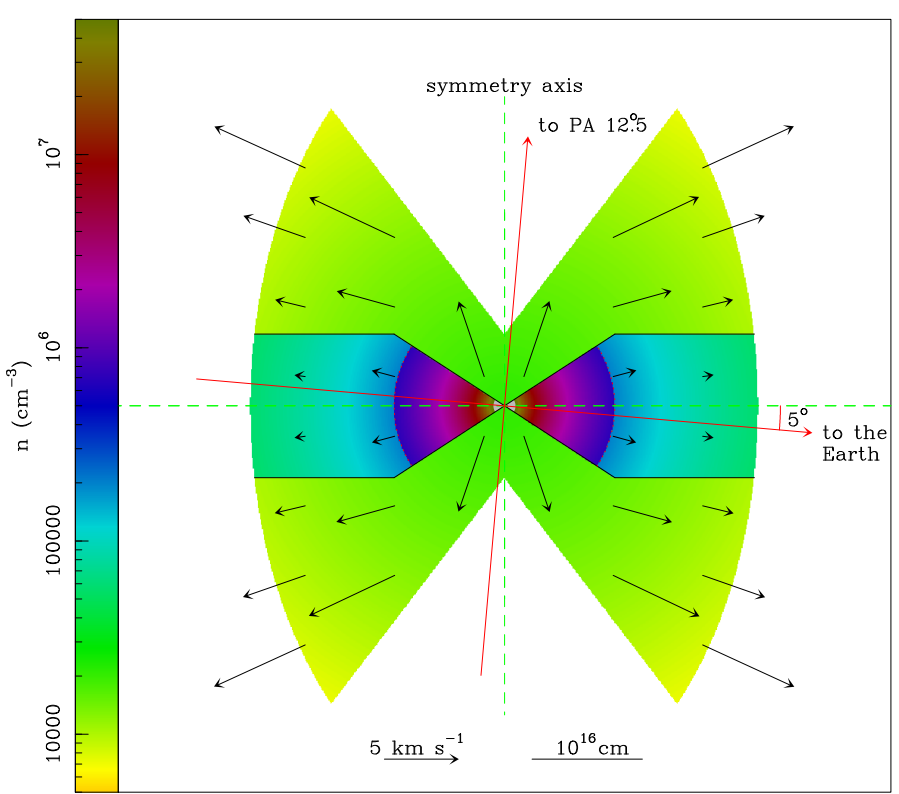

Fig. 8. Structure, velocity, and density distribution in our best-fit model disk and outflow. We represent parameters for a plane perpendicular to the equator and containing the line of sight; therefore, the disk is seen edge-on and only expansion is shown. The velocity and size scales are given at the bottom of the figure.

${ }^{12} \mathrm{CO} J=6-5$, which shows an $\mathrm{X}$-shaped component that is wider than observed, and in ${ }^{13} \mathrm{CO} J=3-2$. This could be the result of a decrease of the $\mathrm{CO}$ abundance from the disk to the outflow, perhaps more important for ${ }^{13} \mathrm{CO}$, which is possible in view of lower density and higher exposure to UV of the expanding component. Nevertheless, the wings of the single-dish data, which come from the outflow, tend to be underestimated in our fitting (Fig. 7, green arrows). Any attempt to obtain weaker outflow emission in the theoretical maps, for instance decreasing the density or abundance in this component, affects the angleintegrated profile, leading to a larger underestimate of the wings of the single-dish profiles. On the other hand, we expect a significant loss of flux in the observed maps at relatively high velocities $\left( \pm 4-7 \mathrm{~km} \mathrm{~s}^{-1}\right.$, Sect. 2.3) due to over-resolution. We conclude that the reason for the overestimate of the outflow emission in our map simulations is probably the systematic loss of flux in the observed maps. In these maps, the weak and extended emission from the outflow, which is relatively more important at high velocities, could be over-resolved. We think that the present fitting is a good compromise between the fact that we know that we are losing flux in the interferometric maps and that we must use them to derive the geometry of the CO-rich nebula. We stress that the steep logarithmic scale we are using tends to give a false, too pessimistic impression of the brightness overestimate of the synthetic maps. Notably, the predicted brightness excess for the outflow in ${ }^{13} \mathrm{CO} J=3-2$, shown in Figs. 1 and 2, affects in practice the very low first contour (at most the second one), at a level that is about 20-50 times weaker than the peak, and has a moderate impact on the single-dish profiles.

The $\mathrm{C}^{17} \mathrm{O} J=6-5$ maps are well reproduced by our model. Section 2.3 shows that the percentage of lost flux is probably much smaller in the maps of the very compact $\mathrm{C}^{17} \mathrm{O}$ (and $\mathrm{H}^{13} \mathrm{CN}$ ) emission. A small contribution from the outflow is seen in both the observed and predicted maps. The model calculations confirm that the weak $\mathrm{C}^{17} \mathrm{O} J=6-5$ line is optically thin. Because of its low opacity and high excitation, this line is a 
good probe of the very inner regions. We used these properties to check the strong increase in density suggested by Men'shchikov et al. (2002) to explain the IR and mm-wave dust emission from this source. These authors proposed an increase of the density by a factor $\gtrsim 10^{3}$ in regions closer than $60 \mathrm{AU}$. In outer regions, the densities proposed in that paper are similar to those found here. However, such a density increase leads to a significant increase of the predicted emission at velocities expected for dense inner regions $\left(\gtrsim 4 \mathrm{~km} \mathrm{~s}^{-1}\right)$; this is in contrast to our observations that show a decrease in the flux at those velocities. Our observations thus do not support a much higher density in the central regions. See some of our tests in Figs. B.3 and B.4.

\subsection{Fitting of single-dish observations of $\mathrm{CO}$ emission}

We also tried to reproduce single-dish mm-wave and FIR observations of the Red Rectangle; see Sect. 2.2. These observations were obtained with the 30-m IRAM radio telescope and Herschel/HIFI; see further discussion in Bujarrabal \& Alcolea (2013). The representation and fitting procedures for these observations are the same as in that paper: the red points are the theoretical results and the black lines are the observed spectra. In the comparison of the observations and the predictions from our best-fit model (Fig. 7), we included and include now a free scale parameter (applied to the predictions) accounting for the calibration uncertainty of the data, which is indicated in percentage in the upper right corner of each of the panels.

The fitting is reasonably good, requiring moderate scaling factors that are compatible with calibration uncertainties. There is a systematic underestimate of the line wings that come from the (relatively fast) outflow; see green arrows in the figure. These underestimates appear in spite of the trend to overestimate the outflow emission in the synthetic maps; this is probably due to a certain degree of over-resolved flux in the interferometric maps of this component (Sect. 5.2); we do not try to improve in order to prevent a worse fitting of the maps.

\subsection{Models of $H^{13} \mathrm{CN}$ emission}

Our maps of $\mathrm{H}^{13} \mathrm{CN} J=4-3$ show weak emission, with a peak of about $1 \mathrm{~K}$, which is clearly indicative of very optically thin emission in comparison with the brightness levels of other lines. The dependence of the emission on the LSR velocity is very different from that found in $\mathrm{CO}$ lines. $\mathrm{H}^{13} \mathrm{CN} J=4-3$ clearly shows intense emission at velocities of $\pm 5 \mathrm{~km} \mathrm{~s}^{-1}$, which are not found in $\mathrm{C}^{17} \mathrm{O} J=6-5$; see Figs. 3 and 5 (see also Fig. A.2). We tried to reproduce this behavior using the same model as for $\mathrm{CO}$, and it has been found to be impossible (Appendix B, Fig. B.5) unless we introduce strong changes in the $\mathrm{HCN}$ abundance depending on the distance to the center. The best fitting is obtained when we assume that $\mathrm{H}^{13} \mathrm{CN}$ is abundant with a reasonable value of its relative abundance $X\left(\mathrm{H}^{13} \mathrm{CN}\right) \sim 10^{-9}$ only in regions closer than $60 \mathrm{AU}$ (drawn in gray in Fig. 8), which are precisely those in which Men'shchikov et al. (2002) proposed a very strong increase of the density; see Sect. 5.1. Otherwise, emission from outer regions at lower velocities largely dominates in the maps (as actually happens for the optically thin emission of $\mathrm{C}^{17} \mathrm{O}$ ).

The significant increase of the $\mathrm{H}^{13} \mathrm{CN}$ could be due to the development of a PDR in dense disk regions close to the central stellar system. The stellar component of the Red Rectangle is a binary system with a secondary star and an accretion disk emitting in the UV (Witt et al. 2009; Thomas et al. 2013). This UV emission is known to be able to excite a central HII region (e.g., Jura et al. 1997) and, therefore, must also yield a PDR intermediate between the HII region and the extended CO-rich disk. Photoinduced formation of $\mathrm{HCN}$ has been observed in inner regions of several disks around young stars and is predicted by theoretical modeling of the PDR chemistry, as a result of reactions triggered by photodissociation of stable molecules in dense PDRs (e.g., Agúndez et al. 2008; Fuente et al. 2012). The presence of photoinduced chemistry is independently supported by observations of the $\mathrm{C}_{2}$ and $\mathrm{CH}^{+}$radicals in our source (see Wehres et al. 2010; Balm \& Jura 1992) and is convincingly confirmed by our single-dish Herschel/HIFI observations of CII and CI FIR lines (Fig. 9). CII emission is thought to originate in PDRs and is in fact the most reliable tracer of these regions in interstellar and circumstellar nebulae, although this emission is less clearly associated with high densities than the production of HCN. CI is often associated with both CO-rich gas and PDRs. As we can see in Fig. 9, the CII line profile shows two components: a wide and intense wing that is very similar to the $\mathrm{H}^{13} \mathrm{CN}$ profiles and a central narrow feature. The origin of the central feature is uncertain; it could come from slowly rotating regions (perhaps the disk boundary exposed to UV photons) or from the very inner outflow (in which axial velocity dominates). The wide wings certainly come from the same region as the $\mathrm{H}^{13} \mathrm{CN}$ emission and are very probably tracing the PDR developed in the very inner disk. The CI $J=2-1$ profile is very similar to the CII profile; in the $J=1-0$ line the central feature seems to dominate, but this line is very noisy and any conclusion is uncertain. The upper panel of Fig. 9 shows the difference with respect to the $\mathrm{C}^{17} \mathrm{O}$ emission, which obviously comes from disk regions less close to the star, in spite of the high excitation required to produce $J=6-5$ emission.

A very low $X\left(\mathrm{H}^{13} \mathrm{CN}\right)$ in the entire disk and a sharp, very strong increase of the density in the inner regions (as that mentioned in Sect. 5.1) can explain the $\mathrm{H}^{13} \mathrm{CN} J=4-3$ data, but (as also mentioned in Sect. 5.1) the results would not be compatible with $\mathrm{C}^{17} \mathrm{O} J=6-5$ maps unless we assume that $\mathrm{CO}$ abundances decrease very strongly in such inner regions. $\mathrm{CO}$ is often found to be depleted onto grains in disks around young stars, but only for very low temperatures and not in warm regions as the central disk of the Red Rectangle. On the other hand, we proposed that $\mathrm{H}^{13} \mathrm{CN}$ is abundant in inner regions precisely because a PDR is developing in them and $\mathrm{CO}$ is being photodissociated. If we assume a significant photodissociation of $\mathrm{CO}$ in inner regions, models with an increase of the density at distances shorter than about $60 \mathrm{AU}$ could then yield acceptable $\mathrm{C}^{17} \mathrm{O}$ emission. However, assuming complementary variations of the $\mathrm{C}^{17} \mathrm{O}$ abundance and the density, by several orders of magnitude, seems to us too ad hoc and difficult to understand. We accordingly think that this option is not acceptable without a significant overabundance of $\mathrm{HCN}$ owing to the presence of a central PDR. We cannot rule out the presence of significant $\mathrm{CO}$ photodissociation in such inner regions in such a way that the emission of $\mathrm{CO}$ isotopologues from them becomes weaker than for normal abundances. This complementary behavior of the $\mathrm{H}^{13} \mathrm{CN}$ and $\mathrm{C}^{17} \mathrm{O}$ chemistry in the inner disk regions would be compatible with the different behavior of the observed $\mathrm{C}^{17} \mathrm{O}$ and $\mathrm{H}^{13} \mathrm{CN}$ emission.

\subsection{Uncertainties in the model fitting}

The number of parameters defining our model nebula is very large and, in fact, not well defined, because the shape and the distributions of the velocity, temperature, and density could admit a high variety of laws. In addition, the amount of data to be fitted is enormous, with maps of several lines for a high number 
V. Bujarrabal et al.: Further ALMA observations and detailed modeling of the Red Rectangle

Table 1. Structure and physical conditions in the molecular disk in the Red Rectangle derived from our model fitting of the CO data.

\begin{tabular}{|c|c|c|c|c|c|c|c|}
\hline \multirow[b]{2}{*}{ Parameter } & \multicolumn{2}{|c|}{ Inner disk $\left(r<R_{\text {kep }}\right)$} & \multicolumn{3}{|c|}{ Outer disk ( $\left.r>R_{\mathrm{kep}}\right)$} & \multicolumn{2}{|c|}{ Outflow } \\
\hline & Law & Values & Law & & Values & Law & Values \\
\hline Outer radius & & $R_{\mathrm{kep}}=10^{6} \mathrm{~cm}$ & \multicolumn{3}{|c|}{$R_{\mathrm{out}}=2.3 \times 10^{16} \mathrm{~cm}$} & & \\
\hline Thickness & linear & $\begin{array}{c}H\left(R_{\mathrm{kep}}\right)=1.3 \times 10^{16} \mathrm{~cm} \\
H(0)=6.5 \times 10^{15} \mathrm{~cm}\end{array}$ & constant & \multicolumn{2}{|c|}{$H=1.3 \times 10^{16} \mathrm{~cm}$} & $\begin{array}{l}\text { ellipsoidal } \\
\text { (see Fig. 8) }\end{array}$ & \\
\hline $\begin{array}{l}\text { Tangential } \\
\text { velocity }\end{array}$ & $\begin{array}{l}V_{t} \propto 1 / \sqrt{r} \\
\text { (Keplerian) }\end{array}$ & $\begin{array}{c}V_{t}\left(R_{\mathrm{kep}}\right)=1.5 \mathrm{~km} \mathrm{~s}^{-1} \\
\left(\text { central mass: } 1.7 M_{\odot}\right)\end{array}$ & \multicolumn{2}{|c|}{$\begin{array}{c}V_{t} \propto 1 / r \\
\text { (ang. mom. cons.) }\end{array}$} & $V_{t}\left(R_{\mathrm{kep}}\right)=1.5 \mathrm{~km} \mathrm{~s}^{-1}$ & & \\
\hline $\begin{array}{l}\text { Expansion } \\
\text { velocity }\end{array}$ & & $0 \mathrm{~km} \mathrm{~s}^{-1}$ & $V_{\exp } \propto \sqrt{a+b /}$ & \multicolumn{2}{|c|}{$\begin{array}{c}V_{\text {exp }}\left(R_{\text {kep }}\right)=1.6 \mathrm{~km} \mathrm{~s}^{-1} \\
V_{\text {exp }}\left(R_{\text {out }}\right)=0 \mathrm{~km} \mathrm{~s}^{-1}\end{array}$} & $|V| \propto \frac{R_{\text {out }}-p_{\mathrm{c}}}{R_{\text {out }}}+\frac{h-H / 2}{310^{16}}$ & $\left|V_{\max }\right|=12 \mathrm{~km} \mathrm{~s}^{-1}$ \\
\hline Temperature & $T \propto 1 / r^{\alpha_{T}}$ & $\begin{array}{c}T\left(R_{\mathrm{kep}}\right)=80 \mathrm{~K} \\
\alpha_{T}=1\end{array}$ & $T \propto 1 / r^{\alpha_{T}}$ & \multicolumn{2}{|c|}{$\begin{array}{c}T\left(R_{\mathrm{kep}}\right)=95 \mathrm{~K} \\
\alpha_{T}=0.6\end{array}$} & $T \propto\left(\frac{R_{\text {kep }}}{p_{\mathrm{c}}-R_{\text {kep }}}\right)^{\alpha_{T}}$ & $\begin{array}{c}T_{\max }=500 \mathrm{~K} \\
\alpha_{T}=1\end{array}$ \\
\hline Gas density & $n \propto 1 / r^{\alpha_{n}}$ & $\begin{array}{c}n\left(R_{\mathrm{kep}}\right)=7 \times 10^{5} \mathrm{~cm}^{-3} \\
\alpha_{n}=2\end{array}$ & $n \propto 1 / r^{\alpha_{n}}$ & \multicolumn{2}{|c|}{$\begin{array}{c}n\left(R_{\mathrm{kep}}\right)=2 \times 10^{5} \mathrm{~cm}^{-3} \\
\alpha_{n}=1.5\end{array}$} & $3 n \propto\left(\frac{6.4 \times 10^{16}-r+R_{\mathrm{kep}}}{6.4 \times 10^{16}}\right)^{\alpha_{n}}$ & $\begin{array}{c}n\left(R_{\mathrm{kep}}\right)=1.6 \times 10^{4} \mathrm{~cm}^{-3} \\
\alpha_{n}=2\end{array}$ \\
\hline \multicolumn{4}{|c|}{ Other parameters } & Law & Values & Comments & \\
\hline & \multicolumn{3}{|c|}{ Axis inclination from the plane of the sky } & & $5^{\circ}$ & \multicolumn{2}{|l|}{ from optical and CO data } \\
\hline & \multicolumn{3}{|c|}{ Axis inclination in the plane of the sky (PA) } & & 12.5 & \multicolumn{2}{|l|}{ from optical and CO data } \\
\hline & \multicolumn{3}{|c|}{ Distance } & & $710 \mathrm{pc}$ & \multicolumn{2}{|l|}{ various arguments (Sect. 1) } \\
\hline & \multicolumn{3}{|c|}{$\begin{array}{l}{ }^{12} \mathrm{CO} \text { relative abundance } \\
{ }^{13} \mathrm{CO} \text { relative abundance } \\
\mathrm{C}^{17} \mathrm{O} \text { relative abundance }\end{array}$} & $\begin{array}{l}\text { constant } \\
\text { constant } \\
\text { constant }\end{array}$ & $\begin{array}{c}1.2 \times 10^{-4} \\
1.2 \times 10^{-5} \\
6 \times 10^{-7}\end{array}$ & $\begin{array}{l}\text { this paper } \\
\text { this paper } \\
\text { this paper }\end{array}$ & \\
\hline
\end{tabular}

Notes. The fitting of the $\mathrm{H}^{13} \mathrm{CN}$ line requires the same structure and velocity field but with a peculiar abundance distribution; see Sect. 5.3. The values of the physical conditions depend on three geometrical parameters: the distance to the center, $r$, the distance to the inner conical boundary of the outflow, $p_{\mathrm{c}}$, and the distance to the equator, $h$. See Fig. 8 for a scheme of the density and velocity distribution.

of velocity channels, as well as single-dish profiles. It is difficult to assign weights to the many observational features, because of the very high dynamic range, complex effects of flux loss, and, in general, various degrees of uncertainty. Therefore, our fitting is just performed by inspection of a high number of predictions and adoption of a solution that is compatible with all observations, taking the instrumental accuracy and various arguments on the probable nebular properties into account. For the same reasons, a mathematical discussion on the uncertainties in our model fitting is very difficult. We just estimate the uncertainties of the main overall properties of the nebula, by looking for variations from our best-fit values that lead to predictions that are incompatible with the data.

The estimate of the disk dynamics is very accurate at a fraction of a km s${ }^{-1}$, since the maps define its rotation very well. The overall expansion velocity is also well estimated with a conservative upper limit to the uncertainty of about $\pm 2 \mathrm{~km} \mathrm{~s}^{-1}$, since the predicted outflow emission velocity is clearly in contradiction with the observed velocity extent for models with larger differences.
We have mentioned that the total extent of the molecule-rich nebula is uncertain, in particular, because of the presence of lost flux in the interferometric data and the lack of sensitivity to detect very weak extended emission. It is probable that the values we give here are a lower limit. Of course, the total size of the nebula is underestimated because certainly it extends farther than the detected emission (Sects. 1, 5.1), at least as a result of photodissociation by interstellar UV photons. The rest of the nebula structure is well defined by our high-resolution observations and is in fact compatible with estimates in our previous papers. The resolution of our maps indicate that the width of the disk, in particular, is accurate within a $30 \%$.

The density and abundance uncertainties are given by the need to match the observed intensities. In view of the significant calibration uncertainties and lost flux, discussed before, we adopt (to estimate uncertainties of our determinations) a conservative limit to acceptable discrepancies of about $50 \%$, which could be lower for certain velocities and lines of sight. In optically thin lines, such as ${ }^{13} \mathrm{CO} J=10-9$ and $J=1-0$ and $\mathrm{C}^{17} \mathrm{O} J=6-5$, the changes in the intensities are roughly proportional to the changes in the abundance. Low- $J$, easily excited 


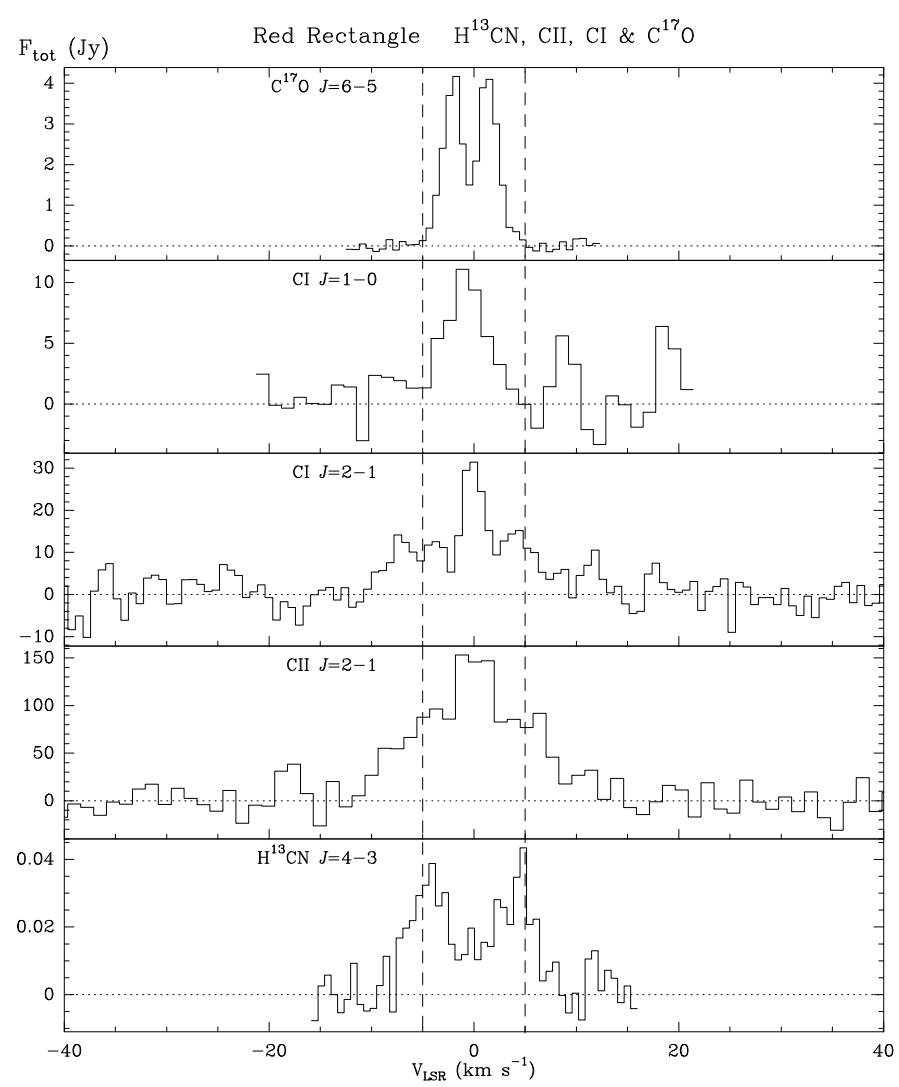

Fig. 9. Observed profiles of the angle-integrated emission of $\mathrm{H}^{13} \mathrm{CN}$ $J=4-3$, CII, CI, and $\mathrm{C}^{17} \mathrm{O} J=4-3$, showing the relative contribution of the central rapidly rotating regions. The vertical dashed lines approximately represent the rotation velocity in regions at about $60 \mathrm{AU}$ from the center; see Sect. 5.3.

CO transitions (say $J<6$ ) show a linear dependence on the density, but high- $J$ lines roughly vary with the square density. Therefore, the effects of both density and abundance cannot compensate when one considers both low- and high- $J$ lines. We conclude (and quantitative calculations confirm this result) that $50 \%$ is a reasonable limit to the uncertainty of our estimates of the overall density and abundance distributions.

We think that the uncertainty in the temperature is lower, because high- $J$ transitions are very sensitive to variations in the temperature. For instance, the synthetic $\mathrm{C}^{17} \mathrm{O} J=6-5$ brightness decreases very strongly if we consider lower temperatures, and $J=16-15$ and $J=10-9$ profiles also vary strongly with the temperature. On the other hand, opaque low- $J$ line intensities (such as ${ }^{12} \mathrm{CO} J=2-1$ ) depend linearly on temperature, but slightly on density. Our calculations show that variations of the characteristic temperature larger than about $\pm 30 \%$ with respect to the best-fitting values are in contradiction with the observations, at least for the rotating disk; for the outflow, uncertainties in the temperature can be higher, $\sim 50 \%$, because of the observational issues affecting this component.

For $\mathrm{H}^{13} \mathrm{CN}$, we assume the same physical conditions deduced for $\mathrm{CO}$. Those maps are relatively noisy and the structure assumed for the very inner regions is not well established. We discussed the extent of the high-abundance inner region in Sect. 5.3: it is clear that $\mathrm{H}^{13} \mathrm{CN}$ cannot be very abundant along the whole disk, but we cannot delimit the size and shape of the inner region rich in $\mathrm{H}^{13} \mathrm{CN}$ with high accuracy. Only an abundance uncertainty of about a factor 2 can be deduced in this case.

\section{Origin of the outflowing gas: tentative rotation of the expanding lobes}

It has been argued that the mass-loss rate of the post-AGB star in the center of the Red Rectangle must be negligible (e.g., van Winckel 2003), therefore, the material that forms the expanding gas detected outside the equatorial disk should come from the disk, which acts as a relatively stable reservoir. Moreover, the outflow structure and velocity deduced from our model fitting strongly support this idea, since we see gas expanding at long distances from the axis and close to the disk, even if most of the expanding gas seems to come from the inner, very dense disk regions. In fact, it is known that the outer parts of the disk shows a slow expansion, together with the obvious rotation (to explain the asymmetry found between the negative and positive velocities in the equatorial regions, see Bujarrabal et al. 2005, 2013b).

If the outflowing gas comes from the disk, it is expected that such a component must share its rotation dynamics at least partially (independent of the dominant expansion). The rotation velocity of the lobes should be very slow because of angular momentum conservation. For radial expansion (which is not necessarily the case here), we would expect a decrease of the velocity proportional to the distance covered during the expansion. For instance, if a particle leaves a region that rotates at, say, $2 \mathrm{~km} \mathrm{~s}^{-1}$, at about $5 \times 10^{15} \mathrm{~cm}$, one expects a rotation velocity of just $0.5 \mathrm{~km} \mathrm{~s}^{-1}$ at a distance of $2 \times 10^{16} \mathrm{~cm}$. Such a field is obviously very difficult to detect, mostly because expansion in those regions reaches a few $\mathrm{km} \mathrm{s}^{-1}$. We are aware of that this reasoning is vague and uncertain because of our poor knowledge of the path followed by the expanding gas, which can be only guessed from the observations. In any case, some rotation should be present in the outflowing gas.

We think that the rotation of the outflow is tentatively detected in our maps. In Fig. 10, we can see position-velocity (P-V) diagrams along various cuts of our ${ }^{12} \mathrm{CO} J=6-5$ image (Bujarrabal et al. 2013b); see also Fig. A.3 for ${ }^{12} \mathrm{CO} J=3-2$. We can see that the cuts H1N and H1S in the figure partially share the rotation pattern seen along the equator (H00). Velocities tend to be slightly positive/negative for positive/negative (eastward/westward) offsets, for both north and south cuts (superposed to the expansion dynamics dominant in these regions). The effect is small, smaller than $1 \mathrm{~km} \mathrm{~s}^{-1}$, in agreement with our discussion in the previous paragraph. As expected, rotation is much less noticeable for cuts $\mathrm{H} 2 \mathrm{~N}$ and $\mathrm{H} 2 \mathrm{~S}$, since the rotation velocities must still be smaller in those cuts, but the asymmetry can still be seen. A weak effect is also present in cuts that are parallel to the axis, cuts V. At distances $\sim \pm 1-1.5$ arcsecond from the equator, the emission is slightly bluer for $\mathrm{VW}$ and redder for VE.

It is improbable that instrumental effects are responsible for these velocity shifts. Contamination of the equatorial P-V distribution through sidelobes is not likely because of the small ALMA beam ( $\sim 0$.' 25 in this case). Moreover, the structure of the equatorial P-V diagram is not reproduced at all in those along the other horizontal cuts, in which rotation is clearly coupled with the expansion signature (the hollow, elliptical features in the P-V diagrams). Contamination by emission of the eventually warped outskirts of the disk is also not likely because in that case one would expect prominent emission at the central offset and velocity. A similar result is found from our maps of ${ }^{12} \mathrm{CO} J=3-2$, Fig. A.3, which show shifts that are almost identical to those seen in $J=6-5$. The very comparable behavior of both $J=3-2$ and $J=6-5$ maps, despite the very different angular resolutions 

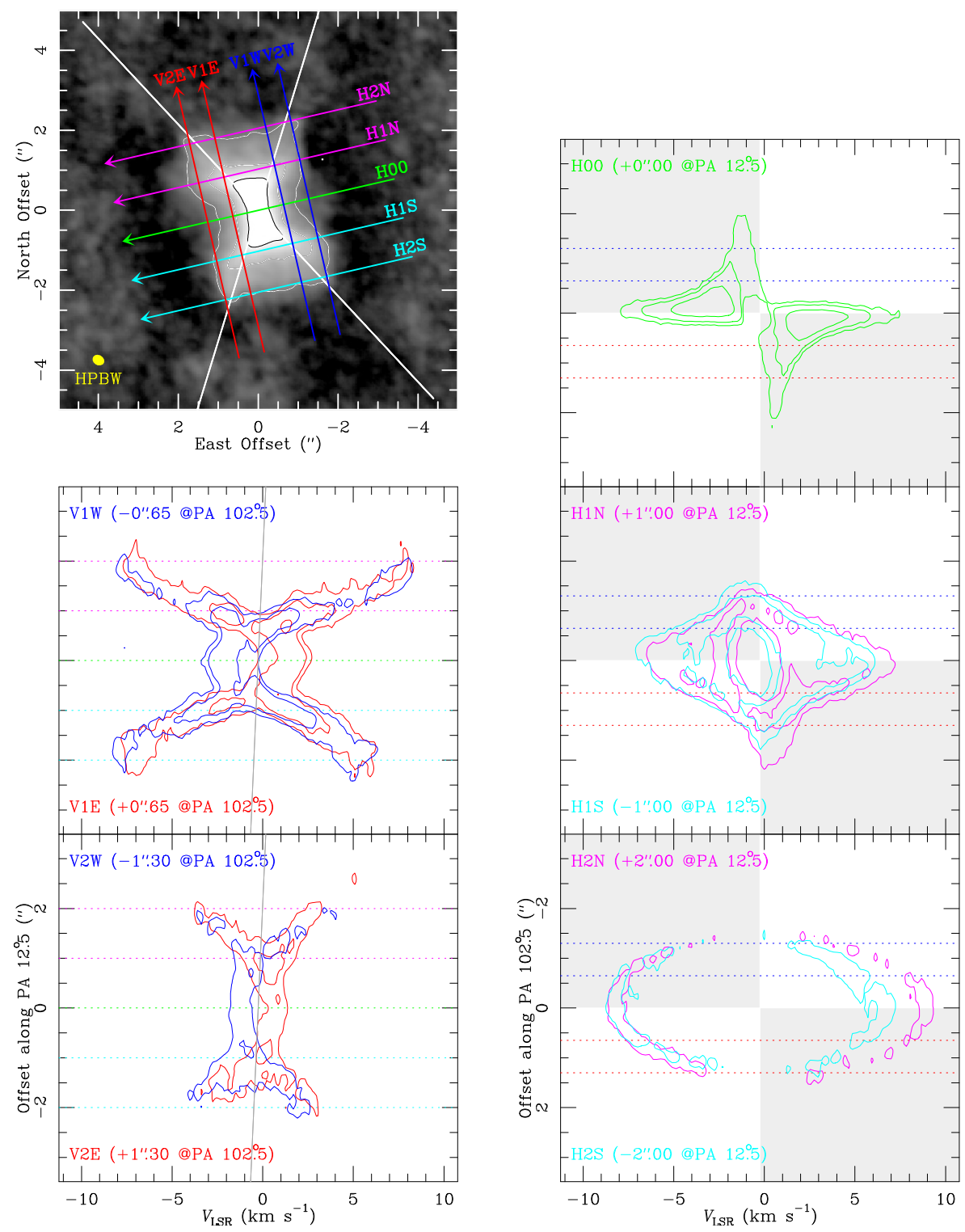

Fig. 10. Position-velocity diagrams of ${ }^{12} \mathrm{CO} J=6-5$ for several cuts, indicated in the upper left panel, which represents the integrated flux map (the arrows indicate the sense of the offsets in the cuts). The cut positions are also indicated, when appropriate, in other panels. For the integrated flux, the first contour is $20 \mathrm{mJy} \mathrm{km} \mathrm{s}^{-1}$, with a logarithmic jump of a factor 3 . For the P-V cuts, the contours are always the same as in the corresponding channel maps. The dark areas in the horizontal cuts indicate the P-V quadrants where we expect that emission tends to concentrate in the case of rotation.

(about a factor 2 better in $J=6-5$ ) and observing conditions, supports that this feature corresponds to true rotation and not to instrumental effects.

\section{Discussion and conclusions}

We present high-quality ALMA maps of ${ }^{12} \mathrm{CO}$ and ${ }^{13} \mathrm{CO} \quad J=3-2$ emission, ${ }^{12} \mathrm{CO}$ and $\mathrm{C}^{17} \mathrm{O} \quad J=6-5$ emission, and of the $\mathrm{H}^{13} \mathrm{CN} J=4-3$ line in the Red Rectangle. The complex structure of the source, which is basically composed of a rotating equatorial disk and a low-collimation bipolar outflow, is confirmed (Sects. 4, 5). The ${ }^{12} \mathrm{CO}$ and ${ }^{13} \mathrm{CO}$ results were already published (Bujarrabal et al. 2013b), but we present here the results of a new reduction of the ${ }^{13} \mathrm{CO} J=3-2$ maps that show emission from the outflow, not obviously seen in our previous publication. We also present Herschel/HIFI observations of $\mathrm{CI}$ and CII line emission from this source, which are compared with the $\mathrm{CO}$ and $\mathrm{H}^{13} \mathrm{CN}$ lines. A detailed model fitting of all the molecular line data, including previously published single-dish observations of several rotational lines of $\mathrm{CO}$, was performed using a sophisticated code that includes accurate nonlocal treatment of radiative transfer (see Sects. 3 and 5); the main properties of the model nebula are summarized in Table 1 and Fig. 8. The existence of high-quality data on low- and high-excitation lines, also showing different degrees of opacity, allows us to study the nebular components in detail. This is the case thanks, in particular, to a more reliable separation of the effects of density and abundance on the calculated intensities (Sect. 5.4).

\subsection{Total mass of the disk and outflow}

From the density and size we derive from our modeling, we find a total mass of the disk of $\sim 1.3 \times 10^{-2} M_{\odot}$, while the mass of the outflow is $\sim 1.2 \times 10^{-3} M_{\odot}$. These values are about two times 
higher than those found from the general and simpler analysis by Bujarrabal et al. (2013a), the discrepancy mainly comes from the lower $\mathrm{CO}$ abundance deduced here, i.e., $X\left({ }^{12} \mathrm{CO}\right) \sim 1.2 \times 10^{-4}$ and $X\left({ }^{13} \mathrm{CO}\right) \sim 1.2 \times 10^{-5}$. These values of the abundance (as well as those used in our previous paper, $2 \times 10^{-4}$ and $2 \times 10^{-5}$, respectively) are compatible with usual estimates of this parameter. The relatively high values of the density and temperature we adopt here are necessary to explain the high excitation found in the disk and outflow, which show intense emission in high- $J$ lines. Therefore, the abundance must at the same time decrease to yield the observed intensities.

The angular momentum of our best-fit model disk is of about $2.7 \times 10^{52} \mathrm{~g} \mathrm{~cm}^{2} \mathrm{~s}^{-1}$, i.e. $\sim 9.1 M_{\odot} \mathrm{AU} \mathrm{km} \mathrm{s}{ }^{-1}$. This value is comparable to (or somewhat smaller than) the angular momentum of a subsolar companion with the orbital period of the Red Rectangle system, which is 320 days. Therefore, if we assume that the disk angular momentum comes from that of the binary system, we conclude that a good deal of the original angular momentum of the system was (in some way) transferred to the disk and that the distance between the stars decreased by a factor of a few to tangentially accelerate the disk material. Nevertheless, the disk of the Red Rectangle seems to be particularly massive and extended, compared with other similar objects for which data exist (Sect. 1), therefore, it is possible that the formation of the Keplerian disks in these other objects have been in general less demanding.

\subsection{Structure and chemistry of the inner Keplerian disk}

Thanks to our high-sensitivity observations of the optically thin, high-excitation lines $\mathrm{C}^{17} \mathrm{O} J=6-5$ and $\mathrm{H}^{13} \mathrm{CN} J=4-3$ we are able to probe the very inner regions of the Keplerian disk. We recall that the well-defined Keplerian dynamics of these regions allows the identification of emission from central regions, rotating at high velocity, even if their extent is at the limit of the telescope resolution.

The $\mathrm{C}^{17} \mathrm{O}$ line is useful to study regions even closer than 60 AU from the central star, which rotate at velocities $\gtrsim 5 \mathrm{~km} \mathrm{~s}^{-1}$. The good fit of the observations with our model shows that the density and temperature of these inner regions still follow the smooth function we find for outer regions (Sect. 5.1). In particular, we do not find any sign of the important increase of the density proposed for such very inner regions from studies of the IR dust emission of the Red Rectangle. Such an increase should have led to very significant emission at velocities farther than \pm $5 \mathrm{~km} \mathrm{~s}^{-1}$, which is not observed. Although CO is a very stable molecule and chemical effects hardly change its abundance, we discuss below (see also Sect. 5.3) that we cannot exclude that photodissociation is significant in the very inner disk regions.

The $\mathrm{H}^{13} \mathrm{CN} J=4-3$ line does show an important emission at such high velocities. The only way to reproduce the data is to assume that $\mathrm{H}^{13} \mathrm{CN}$ is significantly abundant only in the central regions of the disk, precisely closer than 60 AU. We think, following results that are well established in the interstellar medium, that HCN is efficiently formed only in a central PDR (Sect. 5.3). The stellar component of the Red Rectangle is known to emit enough UV to excite a central PDR. See further arguments supporting the presence of photoinduced chemistry in Sect. 5.3, including the detection of CII and CI FIR lines, which are the best tracers of PDRs, that are observed in the Red Rectangle and show a wide spectral feature that corresponds to emission from this inner disk (plus a central narrow feature of uncertain origin).

According to this scenario, we further speculate that PAHs and other C-bearing macromolecules would be efficiently formed and excited because of the strong UV radiation in our source. PAHs are known to be particularly abundant in PDRs and its emission is very intense in them, they are in fact a very good tracer of interstellar PDRs (see Pilleri et al. 2015; Vicente et al. 2013; Cox et al. 2016, and references therein). The formation of PAHs can result from the destruction of grains by UV photons, but they can be also formed by bottom-up chemistry in dense gas if atomic carbon is very abundant due to $\mathrm{CO}$ photodissociation, as has been discussed precisely for the case of O-rich postAGB nebulae by, e.g., Cox et al. (2016) and Guzman-Ramirez et al. (2011, 2014). The X-shaped structure seen in the optical image of the Red Rectangle is known to be emission of PAHs and other (probably carbonaceous) macromolecules; see Sect. 1 and Cohen et al. (2004). We suggest that PAHs may be efficiently formed in the inner disk regions and then expelled with the gas outflow. This result, if confirmed, would be practically independent of the possible change in the dominant chemistry of the star, from O-rich to C-rich (Sect. 1), since PAH emission can be intense even in O-rich PDRs. In Fig. 8, we also show the suggested central PAH factory (the small butterfly-like structure, in gray, with a radius of about $60 \mathrm{AU})$, in which all PAHs seen in the optical would be formed and in which $\mathrm{HCN}$ is much more abundant than in the rest of the disk.

\subsection{Mass loss from the disk and its lifetime}

We have argued (Sects. 1, 6) that the outflowing component detected in the Red Rectangle is probably formed of gas extracted from the disk, in view of the structure and velocity field found in this low-velocity outflow, the possible detection of rotation in it, and some general properties of our sources. The ejection of disk material could be due to interaction with the post-AGB axial jet or low-velocity magnetohydrodynamical ejection of material from the extended disk.

In addition, we derive a decrease in the density between the disk and the outflow by a factor $\sim 10$. With the value of the outflow density close to the boundary of both components and the velocities derived for the expanding gas, and taking the inclination of the velocity with respect to the plane of the disk into account, we can estimate a typical mass-loss rate per surface unit. The value of this parameter at a typical distance to the axis equal to $R_{\text {kep}}$, for instance, is $\dot{M} \sim 1.2 \times 10^{-6} \mathrm{~g} \mathrm{~cm}^{-2} \mathrm{yr}^{-1}$. This rate is of course uncertain, since the velocity field very close to the disk is very difficult to derive from the data. The surface density of the disk at that point is $M \sim 10^{-2} \mathrm{~g} \mathrm{~cm}^{-2}$. Therefore, the (future) lifetime of the disk derived at this typical distance is $t_{\text {disk }} \sim 8000 \mathrm{yr}$. A similar value is found if we compare the total masses of the disk and the outflow taking into account the formation time of the observed outflow of $\sim 1000-1500 \mathrm{yr}$. The outflow lifetime can be derived both from direct inspection of the velocities in our model and from the centroid of the detected expansion in our P-V cuts in the directions V2E, V1E, V1W, and V2W (see the almost vertical gray lines in Figs. 10 and A.3). The presence of outer outflow components not detected in $\mathrm{CO}$ emission, Sect. 1, does not significantly change this conclusion, since a higher total mass would be roughly compensated by a longer outflow lifetime.

The relative mass-loss rates are slightly lower/higher for shorter/longer distances to the center; in the inner regions where rotation is purely Keplerian, which contain about $75 \%$ of the disk mass in our disk model, lifetimes estimated in this way are slightly longer, and more uncertain, since the outflow properties of these very inner outflow regions are poorly probed by our 
data. We must also take into account that the outer parts of the disk are in slow expansion, which should lead to mass loss of the purely Keplerian disk. We estimate a total mass loss of the purely Keplerian regions due to this process of $\sim 10^{-6} M_{\odot} \mathrm{yr}^{-1}$, which, compared to an inner disk mass of $\sim 0.01 M_{\odot}$, would lead to a lifetime of about $10000 \mathrm{yr}$. Therefore, the lifetime of the central regions of the disk is probably comparable or slightly higher than the representative value given above.

We therefore deduce a typical lifetime of the Red Rectangle disk $t_{\text {disk }} \sim 8000 \mathrm{yr}$, probably $\sim 10000 \mathrm{yr}$ for the inner disk regions. We can obtain similar results for other similar NIRexcess post-AGB stars; we stress that they are usually less well studied than the Red Rectangle and therefore the estimates for them are uncertain. In 89 Her (Bujarrabal et al. 2007, 2013a), the mass of both the disk and the outflow are similar at present. The hourglass-like outflow was formed about $3500 \mathrm{yr}$ ago. If the mass-loss rate from the disk does not significantly vary during the post-AGB phases, we can expect a total disk lifetime of about $7000 \mathrm{yr}$, which is smaller than the disk lifetime we found in the Red Rectangle. However, other objects, like AC Her, show a very faint outflow. The outflow was not detected in AC Her in CO $J=2-1$ at a level of about $1 / 15$ of the peak brightness; see Bujarrabal et al. (2015). In the maps of the Red Rectangle obtained by Bujarrabal et al. (2005) for that same transition and with a roughly similar relative resolution, we can see a hint of outflow emission at a level of about $1 / 8$ of the peak. Therefore, the contrast between the densities in the disk and outflow must be $\gtrsim 2$ times lower in AC Her. Assuming the other properties for both nebulae (in particular the outflow velocity and disk width) to be similar, we can conclude that the lifetime of the disk around AC Her is $\gtrsim 2$ times higher than that of the Red Rectangle.

The lifetime of the preplanetary nebula phase is of about $1000 \mathrm{yr}$, as deduced from data of the massive usually observed nebulae. The total lifetime of the planetary nebula phase is usually assumed to be of about $20000 \mathrm{yr}$, after which the shells would be difficult to detect. However, the Red Rectangle and similar objects show a relatively low mass and their central stars are still cool (Bujarrabal et al. 2013a and references therein), so their evolution could be relatively slow. In fact, the Red Rectangle has already spent at least $2000 \mathrm{yr}$ in a post-AGB stage similar to its present one, in view of the extent and velocity of its bipolar nebula. As mentioned before, the hourglass-like nebulae around 89 Her was formed about $3500 \mathrm{yr}$ ago. Therefore, we can expect that the preplanetary nebula phase in these objects lasts significantly more than the above value of $1000 \mathrm{yr}$, characteristic of high-mass preplanetary nebulae.

Given the range of disk lifetimes we derive for our low-mass post-AGB objects, we conclude that the disk mass loss in them, which is supposed to be responsible for the outflowing gas, is the main limit to the Keplerian disk lifetime (at least, to the gas-rich disk lifetime). Therefore, the disk will survive long enough to be detectable during most or all of their post-AGB (preplanetary) phases. We think that at least some of them will survive during the planetary nebula phase provided that the objects we are dealing with here will evolve to form planetary nebulae and white dwarfs following, more or less, our standard ideas on the post-AGB evolution.

Acknowledgements. This work has been supported by the Spanish MICINN, program CONSOLIDER INGENIO 2010, grant "ASTROMOL" (CSD200900038), by the Spanish MINECO, grants AYA2012-32032 and FIS2012-32096, and by the European Research Council (ERC Grant 610256: NANOCOSMOS). We used the Cologne Database for Molecular Spectroscopy, for our discussion on the $\mathrm{H}^{13} \mathrm{CN}$ hyperfine structure, and the LAMBDA database, to obtain the collisional transition rates.

\section{References}

Agúndez, M., Cernicharo, J., \& Goicoechea, J. R. 2008, A\&A, 483, 831 Alcolea, J., Bujarrabal, V., Planesas, P., et al. 2013, A\&A, 559, A93 Balick, B., \& Frank, A. 2002, ARA\&A, 40, 439

Balm, S. P., \& Jura, M. 1992, A\&A, 261, L25

Bujarrabal, V., \& Alcolea, J. 2013, A\&A, 552, A116

Bujarrabal, V., Castro-Carrizo, A., Alcolea, J., \& Sánchez Contreras, C. 2001, A\&A, 377, 868

Bujarrabal, V., Castro-Carrizo, A., Alcolea, J., \& Neri, R. 2005, A\&A, 441, 1031 Bujarrabal, V., van Winckel, H., Neri, R., et al. 2007, A\&A, 468, L45

Bujarrabal, V., Alcolea, J., van Winckel, H., Santander-García, M., \& CastroCarrizo, A. 2013a, A\&A, 557, A104

Bujarrabal, V., Alcolea, J., van Winckel, H., et al. 2013b, A\&A, 557, L11

Bujarrabal, V., Castro-Carrizo, A., Alcolea, J., \& van Winckel, H. 2015, A\&A, 575, L7

Cohen, M., van Winckel, H., Bond, H. E., \& Gull, T. R. 2004, AJ, 127, 2362

Cox, N. L. J., Pilleri, P., Berné, O., Cernicharo, J., \& Joblin, C. 2016, MNRAS, 456, L89

De Beck, E., Decin, L., de Koter, A., et al. 2010, A\&A, 523, A18

de Graauw, T., Helmich, F. P., Phillips, T. G., et al. 2010, A\&A, 518, L6

de Ruyter, S., van Winckel, H., Maas, T., et al. 2006, A\&A, 448, 641

Deroo, P., van Winckel, H., Min, M., et al. 2006, A\&A, 450, 181

Ferreira, J., Dougados, C., \& Cabrit, S. 2006, A\&A, 453, 785

Frank, A., \& Blackman, E. G. 2004, ApJ, 614, 737

Fuente, A., Cernicharo, J., \& Agúndez, M. 2012, ApJ, 754, L6

Gezer, I., van Winckel, H., Bozkurt, Z., et al. 2015, MNRAS, 453, 133

Gielen, C., Bouwman, J., van Winckel, H., et al. 2011, A\&A, 533, A99

Guzman-Ramirez, L., Zijlstra, A. A., Níchuimín, R., et al. 2011, MNRAS, 414, 1667

Guzman-Ramirez, L., Lagadec, E., Jones, D., Zijlstra, A. A., \& Gesicki, K. 2014, MNRAS, 441, 364

Hillen, M., de Vries, B. L., Menu, J., et al. 2015, A\&A, 578, A40

Hillen, M., Kluska, J., Le Bouquin, J.-B., et al. 2016, A\&A, 588, L1

Jura, M., Turner, J., \& Balm, S. P. 1997, ApJ, 474, 741

Mamon, G. A., Glassgold, A. E., \& Huggins, P. J. 1988, ApJ, 328, 797

Soker, N. 2001, ApJ, 558, 157

Panoglou, D., Cabrit, S., Pineau Des Forêts, G., et al. 2012, A\&A, 538, A2

Pilbratt, G. L., Riedinger, J. R., Passvogel, T., et al. 2010, A\&A, 518, L1

Pilleri, P., Joblin, C., Boulanger, F., \& Onaka, T. 2015, A\&A, 577, A16

Sánchez Contreras, C., \& Sahai, R. 2012, ApJS, 203, 16

Santander-García, M., Bujarrabal, V., \& Alcolea, J. 2012, A\&A, 545, A114

Thomas, J. D., Witt, A. N., Aufdenberg, J. P., et al. 2013, MNRAS, 430, 1230

van Winckel, H. 2003, ARA\&A, 41, 391

van Winckel, H. 2014, The Diffuse Interstellar Bands, IAU Symp. 297, 180

Vicente, S., Berné, O., Tielens, A. G. G. M., et al. 2013, ApJ, 765, L38

Waters, L. B. F. M., Cami, J., de Jong, T., et al. 1998, Nature, 391, 868

Wehres, N., Romanzin, C., Linnartz, H., van Winckel, H., \& Tielens, A. G. G. M. 2010, A\&A, 518, A36

Witt, A. N., Vijh, U. P., Hobbs, L. M., et al. 2009, ApJ, 693, 1946 


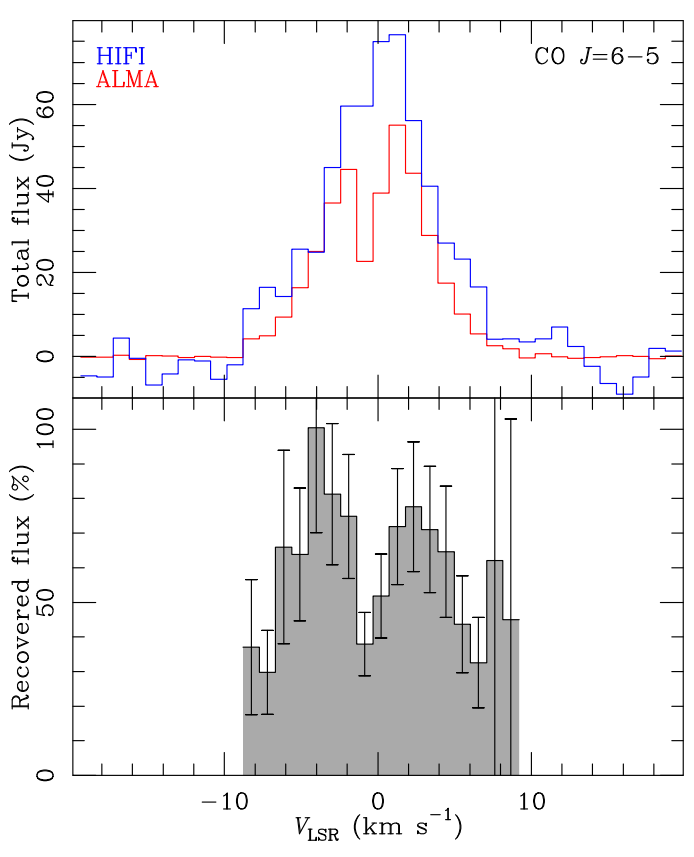

Fig. A.1. Comparison of the ${ }^{12} \mathrm{CO} J=6-5$ single-dish profile and the angle-integrated flux detected in our ALMA maps.

\section{Appendix A: Further observational results and details on data analysis}

In the upper panel of Fig. A.1, we show the flux profile observed with Herschel/HIFI (blue histogram) from Bujarrabal \& Alcolea (2013) and the total (angle-integrated) flux detected in our ALMA maps (Bujarrabal et al. 2013b). In the lower panel, we can see the fraction of the flux actually recovered in the interferometric process, which is found to be high, except for the central channel and intermediate velocities $\pm 5-8 \mathrm{~km} \mathrm{~s}^{-1}$.

Figure A. 2 shows cuts along the equator for all the lines detected and mapped with ALMA in the Red Rectangle. We note the difference between the $\mathrm{H}^{13} \mathrm{CN}$ emission, which is proposed to come only from very inner regions in fast rotation, and those of the CO lines, including the optically thin $\mathrm{C}^{17} \mathrm{O} J=6-5$.

Figure A.3 shows P-V diagrams for the different cuts shown in the upper left panel for the ${ }^{12} \mathrm{CO} J=3-2$ line. The asymmetry in the cuts (particularly in the $\mathrm{H} 1 \mathrm{~N}$ and $\mathrm{H} 1 \mathrm{~S}$ ones) tentatively show the presence of rotation in the outflowing gas. The results are very similar to that found in the $J=6-5$ transition, Fig. 10, showing that instrumental effects can hardly be responsible for the observed asymmetry. The dark areas in the horizontal cuts indicate the P-V quadrants where we expect that emission tends to concentrate in the case of rotation.

\section{Appendix B: Further details on model calculations: changes in the properties of the very inner disk}

In Figs. B.1 and B.2, we show the observed maps of ${ }^{12} \mathrm{CO} J=6-5$ (reproduced from Bujarrabal et al. 2013b) and the predictions from our model for this line, respectively.

Figures B.3 and B. 4 show the predicted maps per velocity channel of $\mathrm{C}^{17} \mathrm{O} J=6-5$ for increases by, respectively, factors of 100 and 1000 of the density in disk regions closer than $60 \mathrm{AU}$. A comparison of these figures with our Figs. 3 and 4 shows that such a very dense central region would lead to emission that is too intense at moderate velocities over $\pm 5 \mathrm{~km} \mathrm{~s}^{-1}$.

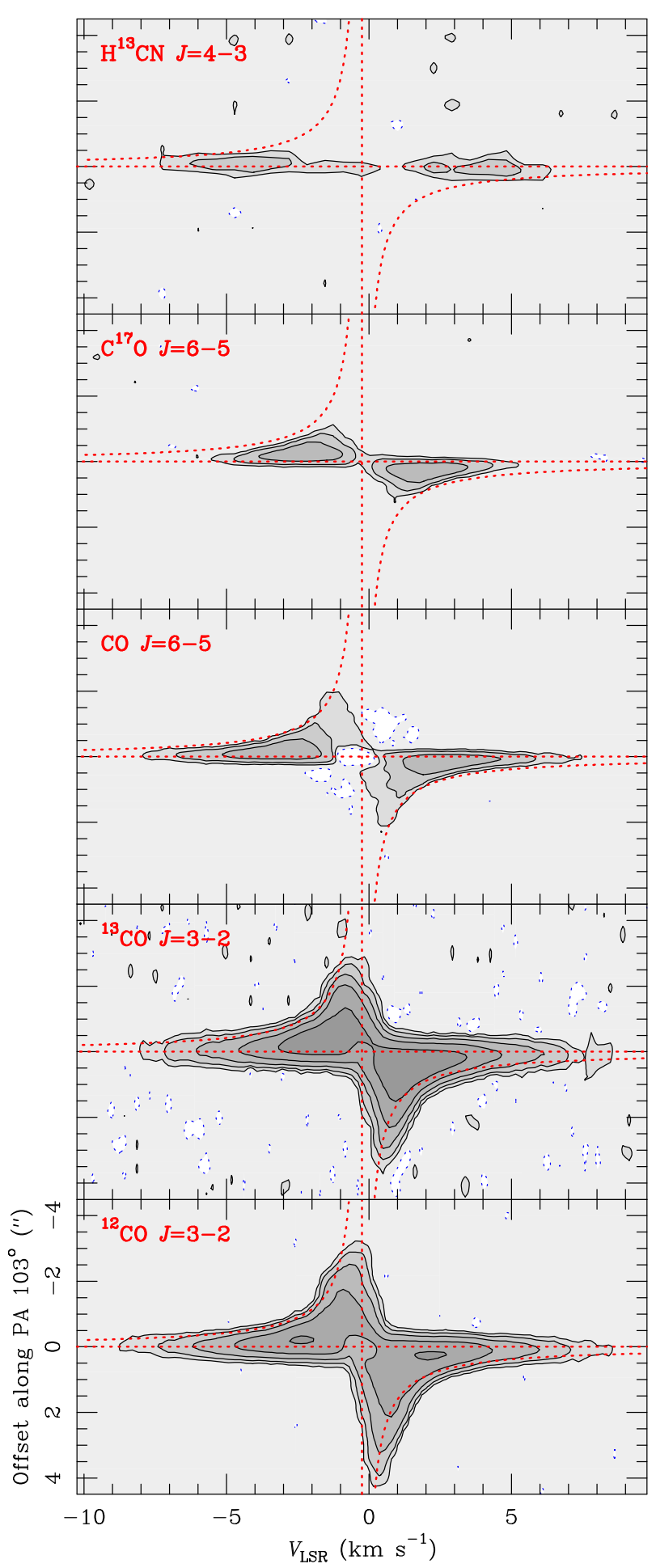

Fig. A.2. Position-velocity diagrams found along the equatorial direction of the lines observed with ALMA in the Red Rectangle. Contours are, as usual, the same as in the corresponding channel maps. To help in the comparison of the different diagrams, we show (red dotted lines) approximate indications of the central position and systemic velocity of the nebula (horizontal and vertical lines) and of the distributions of the emission of gas in Keplerian rotation (hyperbolas).

Figure B.5 shows the expected disk emission in $\mathrm{H}^{13} \mathrm{CN}$ $J=4-3$ emission for a constant $\mathrm{H}^{13} \mathrm{CN}$ relative abundance of $10^{-10}$, for comparison with Figs. 5 and 6 . We can see that predictions cannot match the high $\mathrm{H}^{13} \mathrm{CN}$ emission at moderate velocities. We have chosen a smaller abundance than in our best-fit 
V. Bujarrabal et al.: Further ALMA observations and detailed modeling of the Red Rectangle
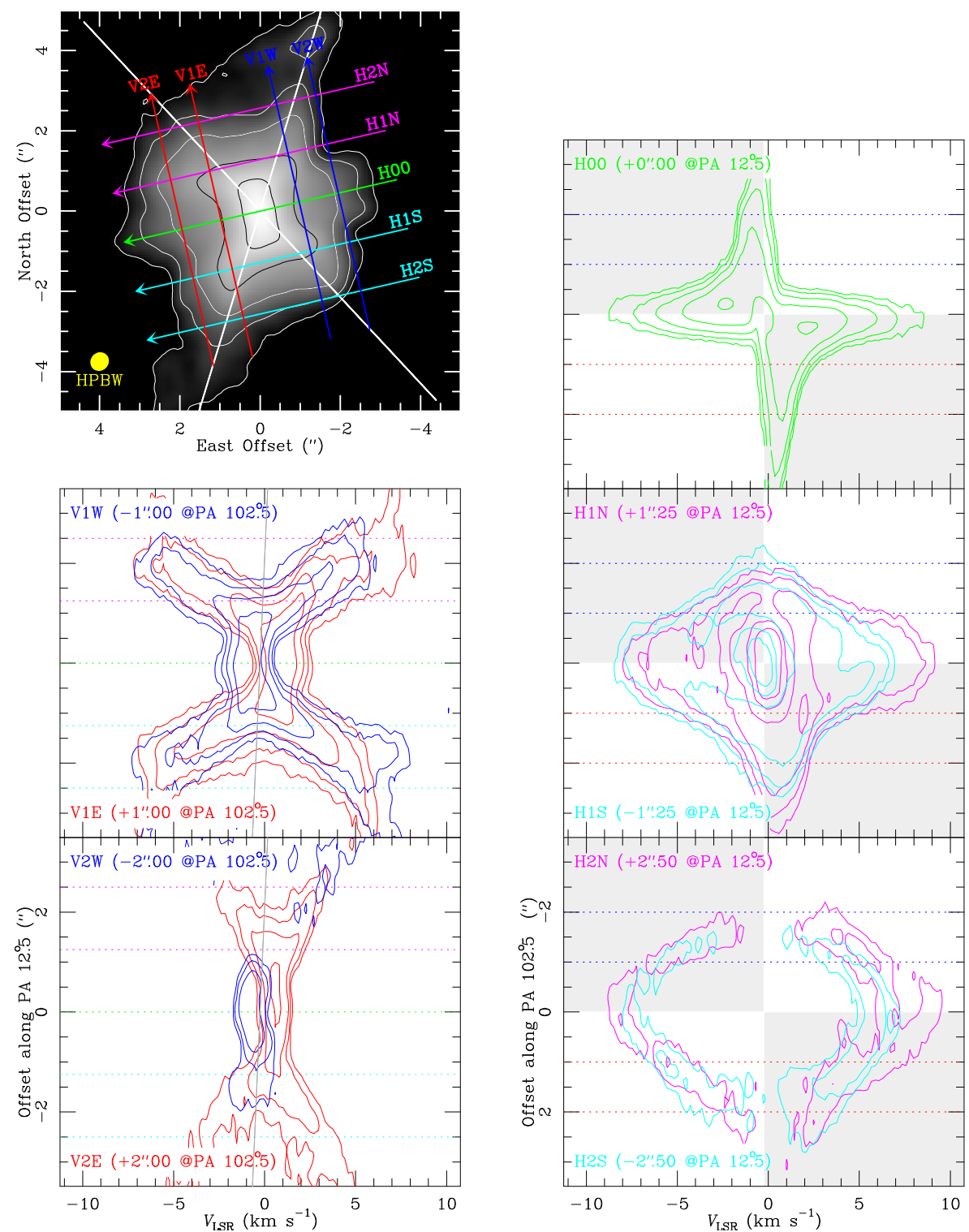

Fig. A.3. Position-velocity (P-V) diagrams of ${ }^{12} \mathrm{CO} J=3-2$ for several cuts, indicated in the upper left panel, which represents the integrated flux map; the arrows indicate the sense of the offsets in the cuts. The cut positions are also indicated, when appropriate, in other panels. For the integrated flux, the first contour is $60 \mathrm{mJy} \mathrm{km} \mathrm{s}^{-1}$ with a logarithmic jump of a factor 3 . For the P-V cuts, the contours are always the same as in the corresponding channel maps. The dark areas in the horizontal cuts indicate the P-V quadrants where we expect that emission tends to concentrate in the case of rotation.

model by a factor 10 because the larger emitting region in the case presented in Fig. B.5 would yield to an emission that is too intense at all velocities and difficult to compare with the observations. 
A\&A 593, A92 (2016)

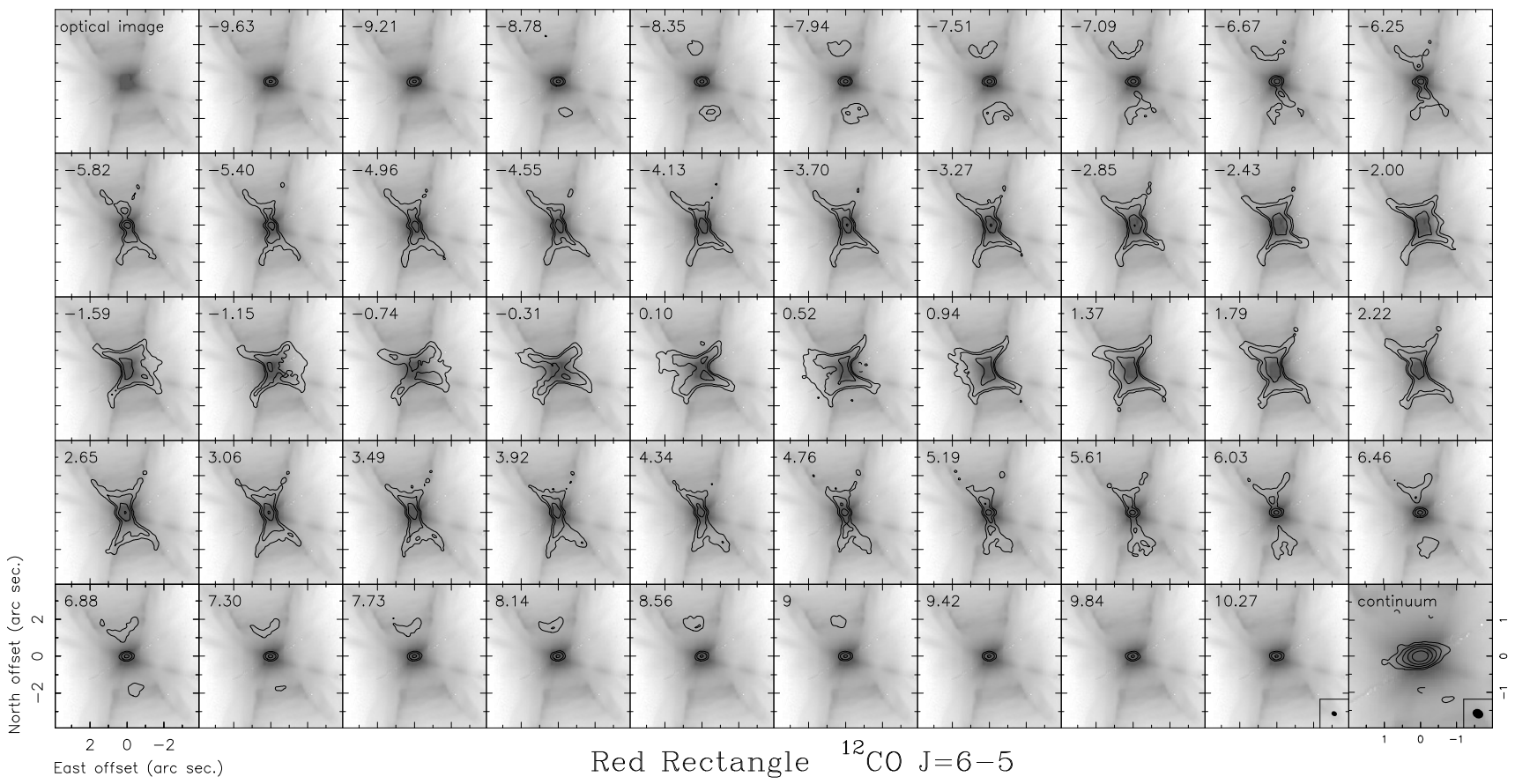

Fig. B.1. ALMA maps of ${ }^{12} \mathrm{CO} J=6-5$, from Bujarrabal et al. (2013b). This figure is reproduced here just to allow comparison with theoretical predictions, Fig. B.2. Continuum is not subtracted in this case because of the intense line emission.

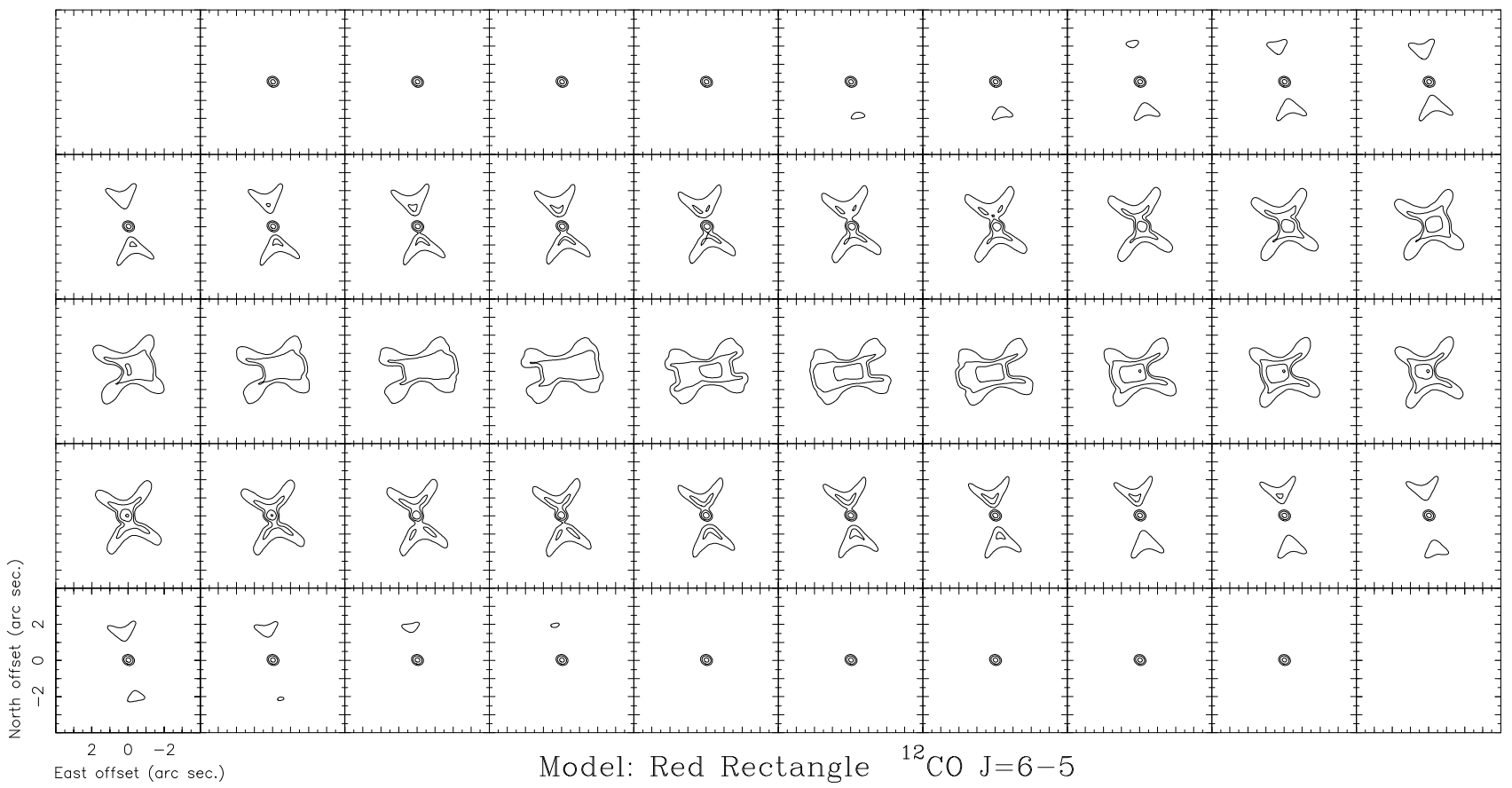

Fig. B.2. Theoretical maps of ${ }^{12} \mathrm{CO} J=6-5$ obtained for our best-fit model, to be compared with ALMA maps published by Bujarrabal et al. (2013b), Fig. 3 in that paper and Fig. B.1 above, in which continuum was not subtracted. The contours and angular and velocity units are the same as in the figure that shows the observational data. 
V. Bujarrabal et al.: Further ALMA observations and detailed modeling of the Red Rectangle

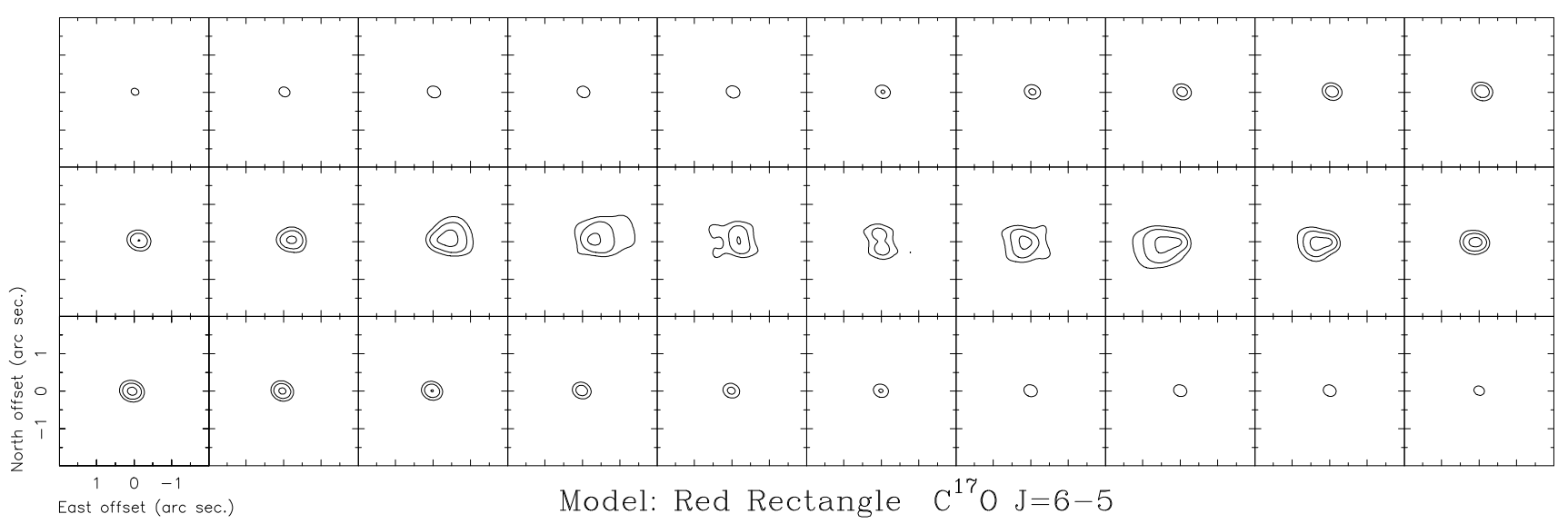

Fig. B.3. Predictions of $\mathrm{C}^{17} \mathrm{O} J=6-5$ maps assuming a conservative increase in the density of the inner disk regions by a factor 100 . To be compared with Figs. 3 and 4.

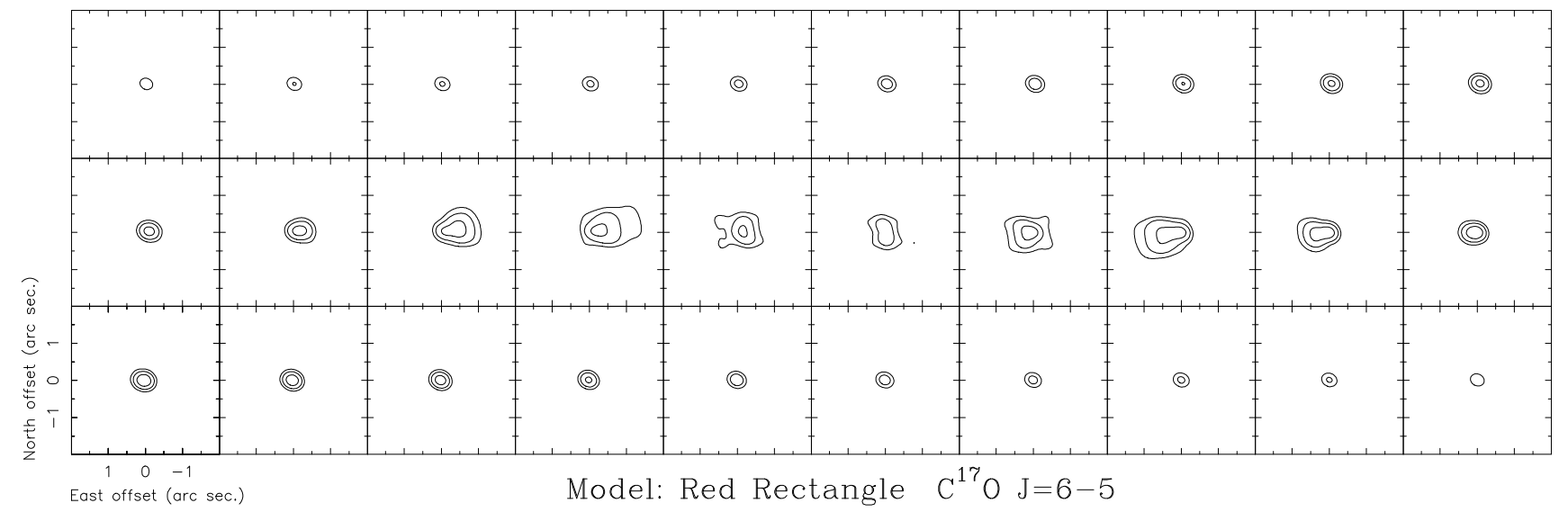

Fig. B.4. Predictions of $\mathrm{C}^{17} \mathrm{O} J=6-5$ maps assuming an increase in the density of the inner disk regions by a factor 1000 . To be compared with Figs. 3 and 4.

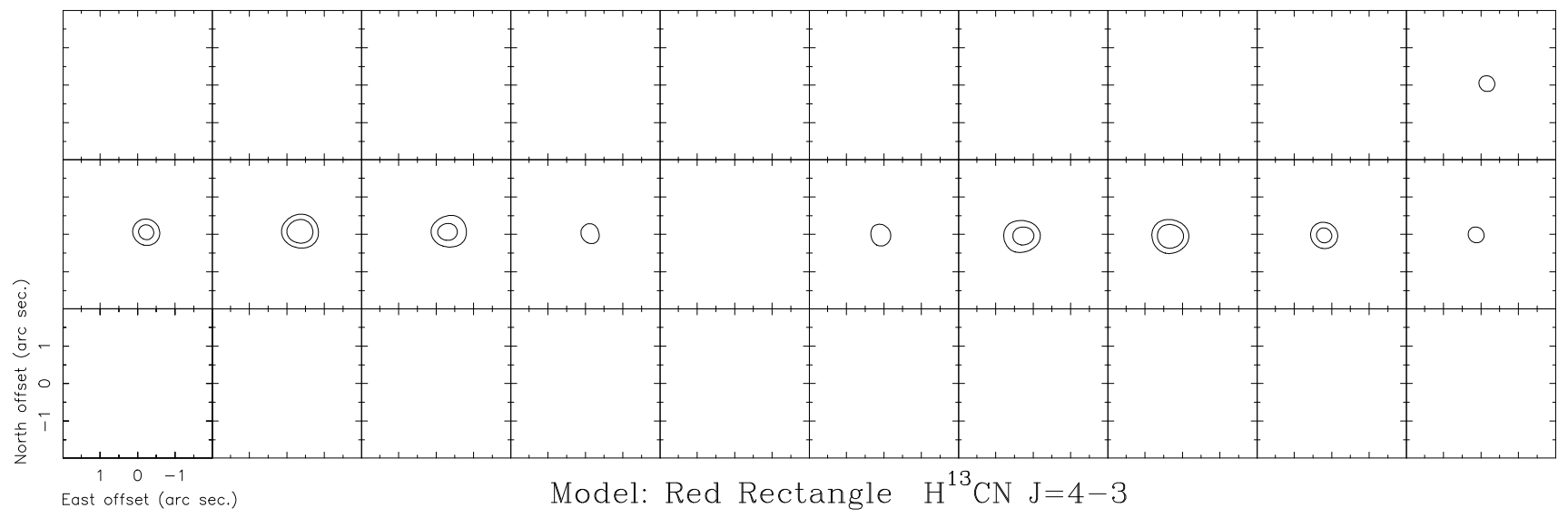

Fig. B.5. Predictions of $\mathrm{H}^{13} \mathrm{CN} J=4-3$ maps assuming a constant abundance across the disk; we took in this case $X\left(\mathrm{H}^{13} \mathrm{CN}\right)=10^{-10}$, which is smaller than in our final model to avoid values of the total intensity that are too strong. To be compared with Figs. 5 and 6. 